Portland State University

PDXScholar

$1-1-2011$

\title{
"You Can See it in Their Eyes:" A Communication Ethnography of a Humane Society
}

Sara Victoria Kaufman

Portland State University

Follow this and additional works at: https://pdxscholar.library.pdx.edu/open_access_etds Let us know how access to this document benefits you.

\section{Recommended Citation}

Kaufman, Sara Victoria, "'You Can See it in Their Eyes:" A Communication Ethnography of a Humane Society" (2011). Dissertations and Theses. Paper 200.

https://doi.org/10.15760/etd.200

This Thesis is brought to you for free and open access. It has been accepted for inclusion in Dissertations and Theses by an authorized administrator of PDXScholar. Please contact us if we can make this document more accessible: pdxscholar@pdx.edu. 
"You Can See it in Their Eyes:" A Communication Ethnography of a Humane Society

by

Sara Victoria Alicia Kaufman

A thesis submitted in partial fulfillment of the requirements for the degree of

Master of Science

in

Communication

Thesis Committee:

Susan B. Poulsen, Chair

Priya Kapoor

Charlotte Schell

Portland State University

(C)2011 


\begin{abstract}
This study sought to understand the culture-sharing group of people working within the shelter area of a Pacific Northwest animal shelter through the Ethnography of Communication. About $63 \%$ of households in the United States live with a companion animal (Risley-Curtis et al., 2006). Recently, there has been a shift toward closer examination into the ways in which humans interact with animals, particularly companion animals.

The guiding questions of this study were: RQ1: What are the cultural communication forms performed in the context of the humane society? RQ2: How do shelter workers communicate about companion animals? RQ3: What cultural meanings are instantiated through communication in this context? This qualitative research approach included 40 hours of participant observation, individual interviews and an analysis of a set of documents and artifacts.

Utilizing the Ethnography of Communication components, thematic \& pattern analysis, findings revealed the use of three main communication forms within the shelter: verbal, written and nonverbal communication, and the overarching key theme of relational bonding occurring within an animal-centric organization among 4 relational categories: A. Shelter animals and shelter animals, B. Shelter workers and shelter animals, C. Shelter workers and shelter workers and D. Shelter workers and the public. Processes leading to relational bonding are delineated including detailed speech as well as aspects of "broken bonds" and euthanasia and its effects within a "no-kill" organization.
\end{abstract}




\section{ACKNOWLEDGMENTS}

It is a pleasure to thank those who made this thesis possible. I want to first thank Dr. Susan Poulsen for her unfailing guidance and support. Dr. Char Schell and Dr. Priya Kapoor provided excellent insights into the findings of this study. I offer thanks to my thesis group partner Jay Lee, who provided encouragement at every step of the process.

I also want to thank the tireless volunteers and staff of the Oregon Humane Society who so generously shared both their time and stories with me. Last but not least I offer my deepest thanks to my family and husband for their support and inspiration. 


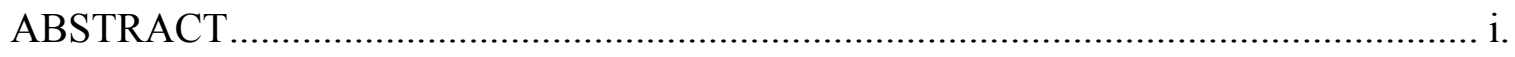

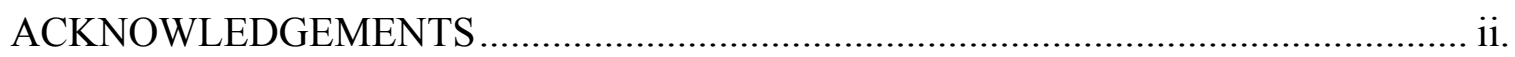
CHAPTER

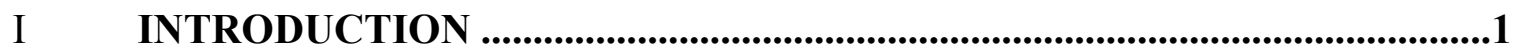

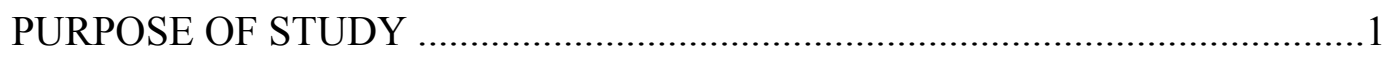

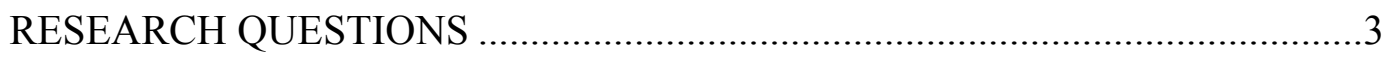

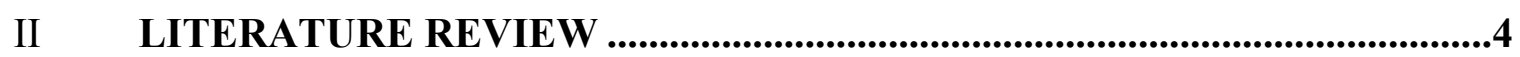

THE HUMANE MOVEMENT IN THE UNITED STATES ..................................

OVERVIEW OF THE OREGON HUMANE SOCIETY ....................................11

III METHODOLOGY AND RESEARCH DESIGN ...............................................12

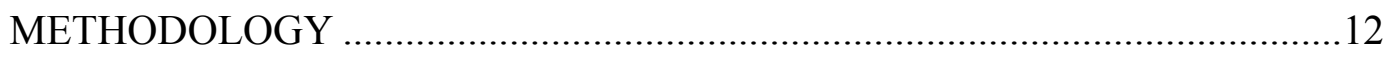

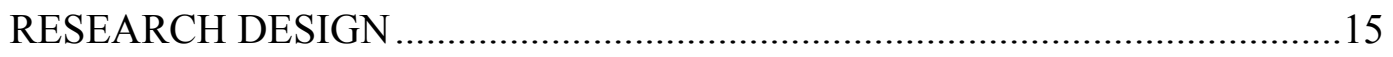

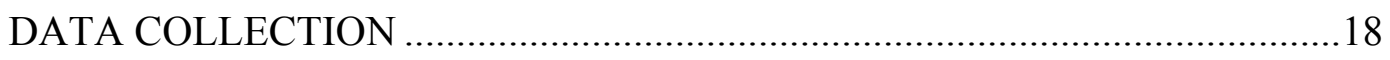

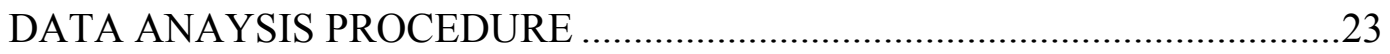

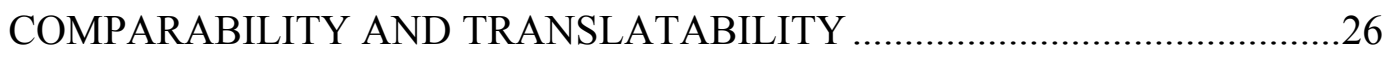

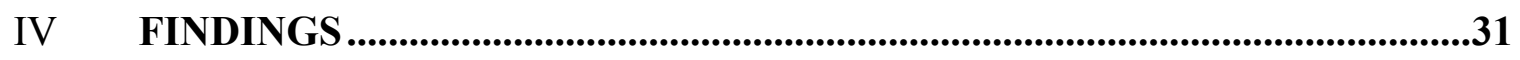

ETHNOGRAPHY OF COMMUNICATION ......................................................

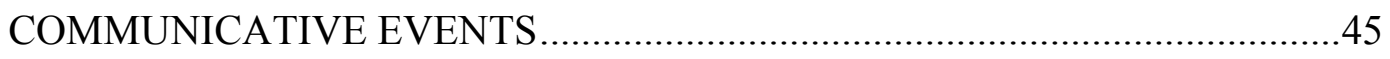

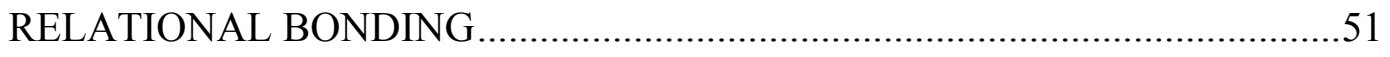


V DISCUSSION AND CONCLUSION

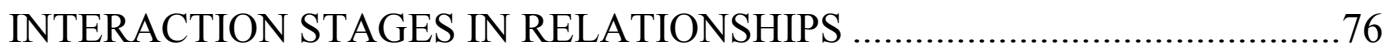

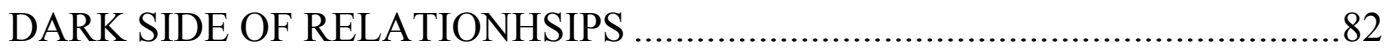

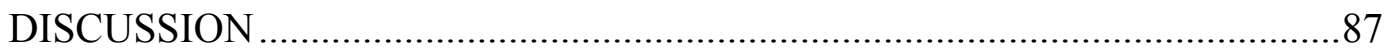

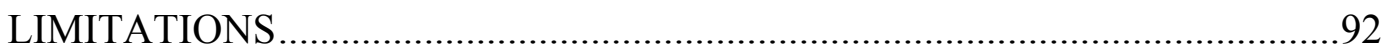

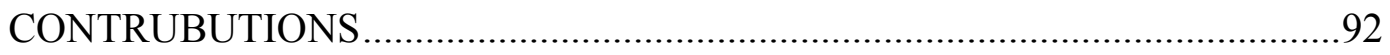

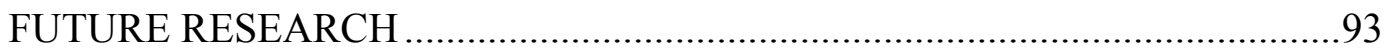

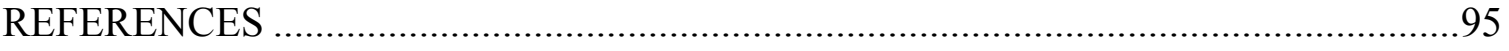

APPENDICIES

A Assumptions and Biases .....................................................................102

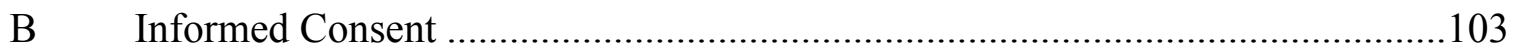

C Letter/Email Requesting Participation.............................................................104

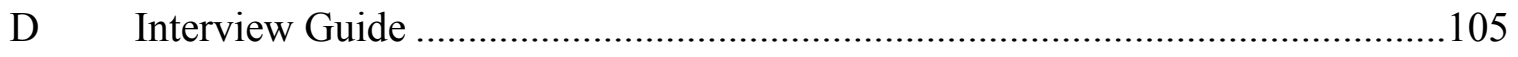

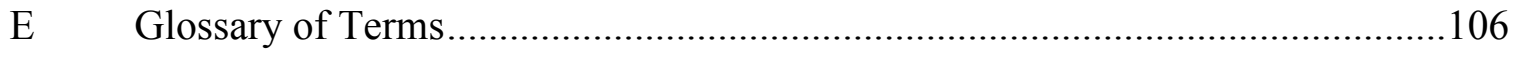

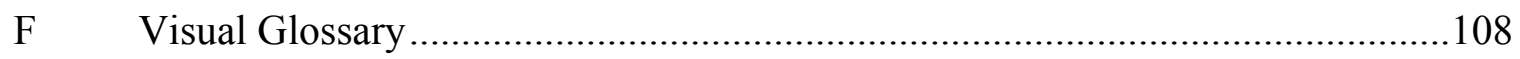




\section{CHAPTER I}

\section{INTRODUCTION}

Understanding the role that companion animals play in human lives is a newly explored field of study within scholarly research (Sanders, 2003). The term companion animal is generally distinguished from the term "pet" to describe domesticated animals, namely dogs, cats, birds and other small mammals that live with humans whose purpose is companionship rather than entertainment or economic function. (Irving, 2004;

Mondelli et al., 2004)

About $63 \%$ of households in the United States live with a companion animal (Risley-Curtis et al., 2006). Recently, there has been a shift toward closer examination into the ways in which humans interact with animals, particularly companion animals. Understanding the way a cultural group utilizes communication within a community can be a significant way to understand their culture. Whereas previous studies have looked at the culture of animal shelters through sociological, psychological, and cultural studies (Furst, 2007; Serpell, 1986; Wolfe, 2009), this study directly addresses communication within the cultural context of a Pacific Northwest animal shelter.

\section{PURPOSE OF STUDY}

The purpose of this study was to understand the culture-sharing group of people working within a Pacific Northwest animal shelter through the Ethnography of Communication. In this study, I focused specifically on the shelter areas of the Oregon Humane Society where volunteers and staff had the most direct contact with animals. 
This study was qualitative and inductive, meaning I was attempting to understand both the meaning and context of this culture's communication by examining the process rather than testing a particular hypothesis.

\section{CONTEXTUAL BACKGROUND FOR THE STUDY}

An interest in companion animals began when my family adopted a cat from a Southern California county animal shelter when I was four years old, and later a dog from a Northern California county shelter when I was in grade school. Our cat lived with our family until he was struck and killed by a car at the age of 17 . Our dog was a young mixed breed that had been picked up from the street and lived with our family for 14 years until she was euthanized due to failing health. I was acutely aware of the role of both shelters in their lives with us. Adopting animals from shelters and living with companion animals appeared to be a part of the typical U.S. experience, one that I had participated in since childhood and throughout my adult life. I was interested in exploring this particular facet of American culture that appeared to be taken-for-granted.

My interest in human-animal interactions continued as I joined the staff in a dog care and boarding facility while working toward my undergraduate degree. As a care provider, I had the opportunity to interact with both the dogs I cared for, and their owners, and observe the interactions between them. Upon moving to Portland Oregon, the Oregon Humane Society appeared as an opportunity to more deeply explore a portion of this cultural community that cared for companion animals. Although I had worked in a setting caring for animals before, I had never volunteered in an animal shelter prior to this study. 


\section{RESEARCH QUESTIONS}

The purpose of this study was to understand the culture-sharing group of people working within a Pacific Northwest animal shelter through the Ethnography of Communication. The guiding questions of this study are: RQ1: What are the cultural communication forms performed in the context of the humane society? RQ2: How do shelter workers communicate about companion animals? RQ3: What cultural meanings are instantiated through communication in this context?

Each research question addresses three main lines of inquiry for this study. The first, (RQ1) will address the communicative forms that comprise speech events, acts and situations (Hymes, 1962). This question will address the structure of communication within the shelter. The second (RQ2) will address the process of how shelter workers communicate. The third (RQ3) research question will address the context or emergent meanings of shelter workers communication.

Chapter II discusses current shelter literature, the history of the humane movement and an overview of the Oregon Humane Society followed by Chapter III, which covers methodology and research design. Chapter IV reports the findings including the Ethnography of Communication while Chapter V discusses the findings as well as limitations and contributions of the study and suggestions for future research. 


\section{CHAPTER II}

\section{LITERATURE REVIEW}

\section{INTRODUCTION}

The purpose of this study was to understand the culture-sharing group of people working within a Pacific Northwest animal shelter. This chapter contains an overview of the history of animal shelters and the humane movement, and discusses past studies of shelter culture.

\section{THE HUMANE MOVEMENT IN THE UNITED STATES}

Within Western culture, humane societies are a fairly recent phenomenon, and are found primarily in communities whose political and socioeconomic environments have advanced beyond "marginal survival" (Arkow, 1985, p. 455).

Legal sanctions against animal abuse to protect animals in transit date back to 1641 within the United States to the Massachusetts Bay Colony. The birth of animal protection as a social movement can be traced to the industrialization and urbanization of the nineteenth century. The 1800 s brought about new knowledge about science and natural history, and with it, social and cultural upheaval. For example, discoveries in astronomy and geology showed that the world was older than was understood, and the universe extended far beyond what could be seen. Evolutionary theory in Darwin's on the Origin of Species (1859) and The Descent of Man (1871) called into question the theological anthropocentrism of the day. Industrialization and mechanized labor was 
replacing animal labor, and animals could now be viewed from a distance, creating curiosity and a new interest and desire to understand them (Franklin, 1994).

Evidence that attitudes were beginning to change regarding the treatment of animals can seen by the number of protection agencies and documented legislation against animal cruelty during this time and "the old way of treating animals ('badly') became increasingly unacceptable to urban sensibilities" (Franklin, 1999, p.12). In 1824, reformers founded the Royal Society for the Prevention of Cruelty to Animals (RSPCA) and other similar animal protection organizations followed in Germany, Austria, Norway and Russia (Arkow, 1999) as well as the Societe protectrice des animaux in France in 1845 (Irving, 2004).

In the United States, the humane movement grew in strength after the Civil War. New Yorker Henry Bergh founded the American Society for the Prevention of Cruelty to Animals (ASPCA) in 1866 after witnessing horses being beat on the city streets (Arkow, 1985) and Massachusetts's attorney George T. Angell petitioned the state to pass general anticruelty laws after having witnessed the death of horses in an 1868 endurance race. Angell was also influential in developing the concept of educating the public about animal cruelty, and promoting humane education (Arkow, 1999; Mason, 2005). In Philadelphia, Elizabeth Morris and Annie Waln began sheltering and finding homes for stray animals picked up from around the city, and in 1874, founded the first Animal Rescue League, which presently operates in Philadelphia as the Morris Animal Refuge (Irving, 2004).

By 1876, over 20 humane groups could be found across the United States (Arkow, 1985). Scholars suggest that various social reforms including the humane 
movement were brought about in the midst of a variety of other shifting public viewpoints that include temperance, abolitionist and anti-vivisection movements (Serpell, 1986). Nineteenth century women in particular became strong advocates within these movements, particularly anti-vivisection linking the medical experimentation of animals and human slavery with their own oppression purported by the mostly male medical establishment and men in general (Finsen \& Finsen, 1994; Buettinger, 1997). Protection of Children

The animal humane movement in the 1800 s became a parent to the child protection movement in both the United States and Briton. During this time children were considered property, and no laws were in place to prevent mistreating them (Arkow, 1985).

The prominent legal 1874 case of "Mary Ellen," a 10-year-old in New York physically abused by her caretakers was brought by ASPCA founder Henry Bergh. In the Supreme Court case, the judge invoked habeas corpus, arguing that Mary Ellen "would be subjected to irreparable harm" if she was not removed from her home (Howard, 2009). The legal case spurred societies for the prevention of cruelty to children (SPCCs) often tied to animal protection organizations (Hall, 1999; Shultz, 1924). Currently, the American Humane Association founded in 1877 is in place today with both animal and child protection divisions (Arkow, 1999).

The origins of The Oregon Humane Society also began through efforts to protect the mistreatment of draft animals and later, the protection of children. The Oregon Humane Society was both Oregon's child and animal protection organization from 1881 to 1933 (OHS, 2008). 


\section{Culture of Shelters}

Previous ethnographic studies have found that staff and volunteers who choose to work in shelters are drawn to the work usually stemming from a desire to work with animals (Alger, 2003, Harbolt, 2003). In one study, shelter employees and staff strongly identify themselves as "animal people" (Arluke, 1994) and view themselves as part of a cultural community engaged in a "battle" against an ignorant public that is the cause of pet overpopulation.

Shelter workers have also viewed themselves as representing the needs of the animals in their care, and drawn to the work due to feelings of disempowerment and victimization. Recent literature suggests that the experience of shelter work often leads to a high level of empathy for animals "based on the perceived experience of shared oppression and suffering" (Harbolt, 2003).

The nature of many U.S. shelters often includes euthanasia, or the killing of animals within the shelter for various reasons, many of which include lack of space within the shelter, behavioral issues of the animals, illness or lack of available homes. The task of performing euthanasia has been shown to take an emotional toll on staff and volunteers who self identify as "animal people," caught in a paradox of being simultaneously viewed both as the rescuers and destroyers of animals in the eyes of the public (Harbolt, 2003).

Studies have shown that volunteers and staff who are charged with performing euthanasia have attempted to manage their emotions by employing various stress management techniques. Some workers focus on ensuring comfort for the animal being killed, or concentrate on successfully completing the technical aspects of euthanasia. 
Others transfer blame onto careless or negligent members of the public, while others attempt to lessen the intensity of their feelings toward shelter animals by adopting a caregiver or social work like role. Workers viewed themselves as engaged in a battle for animals and against an ignorant public. In this way, workers were able to oppose euthanasia at the same time they performed, or assisted in the task (Arluke, 1994).

Another cultural study of the animal shelter environment found that emotional management techniques included resisting attachment to particular animals, substance use and emotional distancing (Harbolt, 2003). Performing euthanasia brought shelter workers into an "inner community," where they did the dirty work created by others but felt criticized by outsiders for killing animals (Arluke, 1991).

Viewing themselves as a cohesive group also helps shelter workers develop community. One ethnographic study of a no-kill animal rescue group found that members constructed narratives in order to redefine the concept of the "humane society" using cultural performance that included distinct metaphors and storytelling that reinforced the group's ideology and culture. These repeated narratives helped to cement the organizational culture despite group members varied backgrounds and outside affiliations (McKay-Semmler, 2007).

\section{Cultural Shift}

Shelters are experiencing a shifting of goals from primarily population control to a focus on retaining animals in new homes and behavioral education (1985, Arkow). Since the early twentieth century, the increased organization of shelters allowed for humane societies to focus efforts on preventing overpopulation of animals and public awareness (Shultz, 1924). 
Many shelters have since transformed from places of "last resort" to centers that integrate a variety of public services that include adoption, sterilization and behavioral counseling. However, due to this shift, shelter workers may find themselves in a position to act as public educator in order to operate within the new conception of shelter culture. Interactional strategies such as emotional regulation and "down time" are suggested in order to successfully communicate information to clients and the public (Irving, 2007).

In light of previous ethnographic studies, there appears to be a gap in the literature which does not fully address the new conception of shelter culture, that is one whose purpose encompasses not only the immediate functions of population control, adoptions and surrenders, but humane education and behavioral training (Irving, 2007). The conception of animal shelters has thus grown in complexity and scope, and there is an opportunity to look at the individuals working within the framework of communication to more fully understand the shared culture of people brought together from a variety of specialties, disciplines and education.

The more researchers understand about this cultural group, the more we will understand about our relationships to and with animals. Irving (2004) states that the way the West relates to companion animals today is fairly recent compared to how long humans have interacted with and used animals. Although people have lived with dogs and cats through the ages, the context of this interaction has been, and continues to be dependent upon religious and sociological norms and expectations. Further, the conception of the need to "shelter" animals from human abuse and neglect, and thus a need for humane societies has been a recent phenomenon, existing in the United States only since 1866 (Arkow, 1985). 
Scholars within the humanities, cultural studies and social sciences have explored a recent interest in Animal Studies as an academic discipline, attempting to shed new light on the ways we view and interact with animals (Wolfe, 2009). Because "the meaning of animals changes across history and across individual lives, revealing not only what we think about animals but also what we think about ourselves, as a culture and individuals" (Arkow, 1985, p.32), understanding the community within animal shelters may allow us to better understand our culture as a whole.

Although previous literature has examined the shelter from primarily a sociological view, the communication of groups whose jobs involve caring for companion animals particularly in shelter settings, is less well documented. The current research does not examine the shelter and its workers as a distinct and separate culture or speech community. Researchers have called for an inquiry into the specific language and communication of shelter workers:

"Language play is an interesting and unique quality of working in these fields, and could provide a rich area for further study, especially as language relates to the categorization of animals and pets...Verbal play, black humor, categorization, talking to and about the animals ("dogese" or "doggeral") relay a great deal of information about how these animals are experienced by the workers" (Harbolt, 2003, p. 82).

Using Ethnography of Communication (EOC), I studied the cultural community of shelter workers through their communication in order to understand how this group communicates about companion animals and what cultural meanings are instantiated through communication in this context. 


\section{OVERVIEW OF THE OREGON HUMANE SOCIETY}

The Oregon Humane Society was established in 1868 as one of the first humane organizations in the country. OHS is a nonprofit agency that operates without government funding, and is funded through voluntary private and public contributions. The organization is presently located on 10-acres of land in North East Portland, in a mostly industrial neighborhood. The shelter adopted out 10,000 animals in 2009, and states that they do not euthanize due to space limitations, only "for significant health or behavior issues" (OHS, 2008). The mission statement of OHS is:

To foster an environment of respect, responsibility, and compassion for all animals through education, legislation, and leadership. To care for the homeless, to defend the abused, and to fight with unrelenting diligence for recognition of the integrity of all animals. (OHS, 2008)

As the third largest shelter in the United States, OHS provides a variety of public services that include sheltering, adoptions, training, humane investigations and humane education outreach (Oregon Humane Society, para. 1, 2008). The shelter also houses a 24-hour veterinary center and participates in numerous community outreach events.

At the time of data collection, OHS total staff and volunteer population at the shelter numbered 1,458. The Oregon Humane Society is a "no kill' animal shelter. No kill is a term that is used for shelters that do not euthanize unless it is considered the "sole humane alternative" available as in the case of terminally ill animals (Alger, 2003).

As OHS is currently the largest shelter in the Portland metro area, there is an opportunity to understand this Portland community as well as the culture of the individuals working with and communicating about companion animals. 


\section{CHAPTER III}

\section{METHODOLOGY AND RESEARCH DESIGN}

\section{INTRODUCTION}

The purpose of this study was to understand the culture-sharing group of people working within a Pacific Northwest animal shelter through the Ethnography of Communication. The guiding questions of this study were:

1) What are the cultural communication forms performed in the context of the humane society? 2) How do shelter workers communicate about companion animals? 3) What cultural meanings are instantiated through communication in this context?

This chapter consists of the following sections: 1) methodology, 2) research design 3) data collection and data analysis procedures, and 4) reliability and validity.

\section{METHODOLOGY}

\section{Qualitative Approach}

This study was conducted using a qualitative research approach. Qualitative research is derived from naturalistic inquiry, in that data are derived from studying the real world and attuned to emergent data (Patton, 2002). The purpose of qualitative inquiry is an "opportunity to glimpse the complicated character, organization and logic of culture" (McCracken, 1988). A qualitative approach to research provides a holistic picture of a culture through data that consists of the view of informants in a natural setting (Creswell, 1998), and seeks to understand how participants make sense of their world and the context within which participants act (Maxwell, 2005). 
Qualitative research was appropriate for this study because the goals of this study are: (RQ1) What are the cultural communication forms performed in the context of the humane society (RQ2) How do shelter workers communicate about companion animals? (RQ3) What cultural meanings are instantiated through communication in this context? As I attempted to understand both the meaning and context of this culture's communication by examining the process rather than testing a particular hypothesis, an inductive, qualitative approach was most appropriate. Ethnography of Communication as a theoretically grounded method reflected the major tenants of qualitative research noted above.

\section{Ethnography of Communication}

Because the conception of the shelter has grown to include many different specializations and knowledge (1985, Arkow), the previous model of shelter culture has evolved and the new model has been thus far unexplored by researchers within the study of communication. Drawing on Dell Hymes (1962), Saville-Troike (2003) and Clifford Geertz (1973) as a model, I employed the Ethnography of Communication as a descriptive theoretical framework to inform this study of the speech community at the Oregon Humane Society.

\section{Culture}

This ethnographic study draws on the concept of culture as identified by Geertz (1973). According to Geertz, humans are "suspended in webs of significance" (p. 5) or their culture ${ }^{1}$. Geertz states that when analyzing culture, that it is not necessary to search for universals, and that patterns of behavior should not be thought of as necessarily

\footnotetext{
${ }^{1}$ Attributed to Max Weber
} 
concrete, but as variables in a larger system, the interpretation of which should be an inductive search for meaning. Cultural patterns of religion, philosophy and aesthetics function as "blueprints" for sociological processes of a culture. Communication and language in particular are one of these major systems, through which ideology itself is transmitted; therefore, looking at the processes and functions of language within a culture is a key to understanding the culture itself.

\section{Language}

Hymes (1962) states that culture is largely transmitted through language, and that language cannot be separated from a theory of human behavior. Hymes proposes The Ethnography of Speaking as a way to understand a community by examining language in the context of situations, uses and patterns. By looking at the structures of language, a cultural community can be understood through discourse.

Similarly, Philipsen (1992) states that the ethnography of speaking is essentially a report on a culture, and rests upon a set of assumptions. Philipsen states that speaking is structured, in that it is organized by grammar and is patterned. Speaking is distinctive, in that speech and language is structured within a context of a distinct social life. Finally Philipsen posits that Speaking is Social, or that speech is not just a medium of social interaction but shapes social life. Speaking unites and ties people to a particular community. Philipsen states that the ability to participate in the social life of a community through speech is in fact "resources for communal identification, and communal being" (Philipsen, p. 14).

The theory that language is closely tied to community is also demonstrated by Saville-Troike (2003) who identifies a community as one that includes aspects of "shared 
knowledge, possessions, or behaviors" (p. 15) which in turn is related to how that group uses language. Further, Saville-Troike states that language provides a way to understand what is important to a community, assumptions about the beliefs, values and rights accorded individuals as well as a way to define and mark social and political categories.

Ethnography of Communication is now understood to include a range of communication behaviors, which include nonverbal communication. Traditional Ethnography of Speaking focused primarily on linguistics, or the verbal code. However, nonverbal communication is generally included as way to understand the speech community (Saville-Troike, 2003). The Ethnography of Communication (EOC) will be used to refer to the methodology and framework of this research study.

\section{RESEARCH DESIGN}

\section{$\underline{\text { Research Site }}$}

Oregon Humane Society is the third largest animal shelter in the United States. Founded in 1880 by Dr. Thomas Lamb Eliot after he witnessed the beating of a carriage horse, OHS was Oregon's child and animal protection organization from 1881 to 1933. Located on 10 acres in an industrial area of North East Portland, the shelter was renovated in 2000 to include new veterinary and educational facilities including Animal Medical Learning Center (AMLC), and increased space in the dog kennels and the cattery (OHS, 2000). The humane society now encompasses a variety of different areas that include veterinary care, adoptions, a behavior department, humane investigations and conducts regular classes for the public as well as internal workshops for staff and volunteers. 


\section{$\underline{\text { Access }}$}

I was initially in contact with the Education Manager at OHS who was my initial contact for the pilot study, and who gave me permission to use the shelter as a research site for the thesis. The manager initially served as a gatekeeper to the site. A gatekeeper in qualitative ethnographic research is a participant in the study who the researcher develops a relationship in order to gain and maintain access to a research site (Maxwell, 2005).

\section{Data Types}

Ethnography of Communication data types include background information, or a history of the population, material artifacts to understand "how a speech community organizes experience in relation to language" (Saville-Troike, p. 93). Social organization or structure, artistic data and common knowledge, or assumptions about language and beliefs about language use, as in who is capable of speech, and positive or negative views surrounding language use. This qualitative ethnographic approach to addressing data included field notes, material artifact analysis and face-to-face interviews.

\section{Field Notes}

I took detailed field notes of my experiences and observations as a part of participant observation during my volunteer time and wrote detailed notes about the experience directly after. Field notes included interactions and experiences with animals, staff, volunteers and the public, often writing while sitting with a dog in their kennel, or sitting with a cat in the cattery. Notes were also made of the sounds, scents and language of the shelter, paying particular attention to patterns of speech and written communication. 
Observations included drawing diagrams of the setting, which included the interior shelter areas of the dog kennel pods, the cattery and small animal rooms as well as the exterior of the building. Photos were also taken of visual communication inside the shelter, such as posters and artwork. I also spent time observing the exterior of the shelter and shelter grounds to understand shelter as it was geographically situated.

As the researcher is used as a tool for information gathering and data analysis in ethnographic research (Patton, 2002), I kept a record of my thoughts and observations and recorded them as either Analytic memos (AM) or Personal Memos (PM) within my observation notes and in a separate file. These memos served the purpose of recording my own thoughts and reflections to facilitate insight and to develop further theoretical ideas (Maxwell, 2005). In addition, I kept detailed reflection notes of my experiences before and during my time in the field. Recording reflections after observing and interviewing can serve as quality control because insights may emerge that might otherwise be forgotten or lost (Patton, 2002)

\section{Material Artifacts \& Documents}

"Material culture" such as documents, records, letters and objects can also be studied as sources of data (Patton, 2002, p. 293). Documents and artifacts can act as "stimulus for paths of inquiry" (p. 294) that can then be followed through direct observation and interviews. Documents can also provide insight when collecting interview and observation data, particularly if the interviews and direct observation provide information that contradicts the documents.

Documents and artifacts are also "stable" sources of data, in that they may both reflect past situations and can be analyzed and reanalyzed. They are rich sources of 
information, in that they appear in the natural language of the setting (Lincoln \& Guba, 1985). Similarly, documents can also provide a historical perspective on the organization or culture and may assist the investigator in understanding the culture of study within the context of historical markers, how the culture may have changed and the stories that are told about the culture's history (Patton, 2002). I analyzed 10 volunteer emails that were sent out over the course of 9 months, a printed packet of handouts for new volunteers, two monthly magazines, one promotional mailer and photographs taken onsite by the researcher.

Interviews

Ethnographic interviews are a primary method for data gathering within Ethnography of Communication in order to acquire a general understanding of the culture under study, as well as an understanding of communicative norms (Briggs 1986). A total of 8 interviews were conducted with volunteers and staff working in the animal shelter areas of OHS.

\section{DATA COLLECTION}

\section{$\underline{\text { Interviews }}$}

Interviews are used within qualitative research to understand an individuals point of view through the description of their own lived experiences (Luborsky, 1994) and can assist in the researcher's understanding of the assumptions and relationships that make up the interview participant's view of the world and the topic under exploration (McCracken, 1988). An interview guide with open-ended questions was constructed 
including prompts to ground the interview while also allowing for further inquiry (see Appendix D).

A pilot interview was conducted with the volunteer coordinator at OHS. Conducting a pilot interview at OHS allowed me to test the questions of my interview guide and later adjust for clarity or new information that may have arisen from the pilot interview. She was selected for the pilot interview because she was a staff member but did not work directly in the shelter area where the study was focused. After completing the pilot interview, I revised my initial interview guide, adding three questions that better addressed communication and language, revised two questions that were awkwardly phrased and added three new open-ended questions that I hoped would elicit narratives from interview participants. The volunteer coordinator also acted as a "gatekeeper" in this study, as she answered additional questions about the shelter. A gatekeeper in qualitative research is a participant in the study who the researcher develops a relationship in order to gain and maintain access to a research site (Maxwell, 2005).

\section{Sample Participants and Size}

This study explored the cultural community of the humane society through two different perspectives: volunteers and staff. In order to understand the culture of people whose jobs involve caring for companion animals, I conducted a total of 8 interviews with adult staff and volunteers who work with, and who are in direct contact with animals at the shelter in their daily work.

Three interview participants were recruited through purposeful, or judgment sampling. The purpose of judgment sampling is to select informants who will be able to provide a rich amount of information, and provide the most information about the culture 
(Patton, 2002). Two of the three taught classes at the shelter I had attended and the third had been my shelter mentor; they trained me in the policies and procedures within the shelter. The remaining five interview participants were recruited through snowball or chain sampling (Patton, 2002) where each interview participant was asked at the conclusion of the interview if they recommended a volunteer or staff member to talk to further about the topic.

$\underline{\text { Interview Process }}$

Recruitment began after approval from the Human Subjects Research Review Committee was received on July 14, 2010. I first contacted the Education Manager first through email and met in person to briefly explain the study and request permission to conduct interviews with volunteer and staff and observe at the shelter. Youth volunteers between the ages of 12-17 participated in volunteer activities at the shelter, but worked under the supervision of youth volunteer coordinator during pre-defined shifts and were easily identified by badge and clothing. As my participant sample excluded this population, these measures ensured that I was recruiting subjects above 18 years of age.

After I received permission from the Education Manager to recruit volunteers and staff, I first contacted the volunteer leader of the TTouch class I had attended and discussed the study with her. She had expressed interest in participating in an interview, and set up the interview at a time and place of her choosing. All volunteers and staff contacted for an interview were sent an email explaining the purpose of the study and a copy of the consent letter to read and review (Appendix B). Once they had agreed to participate in this study, all interviews were conducted in person at a time and location of 
the interview participant's choosing. Interviews were not conducted until the informed consent had been read and signed.

A total of 8 interviews were conducted, with the interviews averaging 56 minutes, or about one hour each. The individual interviews took place in several public locations, all at the interview participants choosing. Two interviews took place at the upstairs OHS administration copy room and a "quiet room" or lounge room at OHS. Five interviews took place in locations offsite at three coffeehouses, one restaurant and one bookstore in and around the Portland Metro area.

The environment of interviews is considered important in ethnographic data collection. I assumed that some interview participants might be uncomfortable talking about sensitive experiences onsite at OHS, and I wanted to ensure that they had a choice in whether to participate in the interview on, or off-site. Therefore, I suggested a coffee shop or other place of their choosing as an alternative to meeting at the shelter. Two participants chose to conduct the interview at the shelter; six chose to conduct the interview off-site. One location, a restaurant was relativity noisy, but the recorder picked up all audio of the interview.

\section{$\underline{\text { Recording }}$}

All interviews were audio-taped with a high quality audio recorder with the participants' knowledge, and written consent.

\section{$\underline{\text { Transcriptions }}$}

All interviews were transcribed by the researcher and typed into a word processing program that included date, start and end time, place and pseudonym. All transcriptions were contained within password-protected files. 


\section{Participant Observation}

Ethnographic qualitative research involves gathering direct observation as data within the naturalistic research setting. Through firsthand experience, the investigator is better able to understand the context of participants' interactions, information that cannot necessarily be captured solely through interviews (Patton, 2002). Participant-observation is a common method of fieldwork in which the researcher attempts to immerse themselves into the speech community in order to separate themselves from their own cultural filters and experiences (Saville-Troike, 2003). Direct observation allows the researcher to observe things that participants may not be aware of or pay attention to, and is also an opportunity to learn things that participants may not reveal in interviews. In addition, observation through firsthand experience allows the researcher to reflect upon personal knowledge and impressions to be used as data.

In order to gain perspective of a shelter worker, I began as a new volunteer and both observed and participated in shelter activities. As a volunteer, I spent a total of 40 hours of participant observation over 9 months in the shelter areas from July 2010 to March 2011. This included 30 hours in the dog shelter area and 10 hours in the cat shelter area. As a volunteer, we were encouraged to choose only one area first. I chose the dog shelter because this was the area I initially had the most comfort and experience with. As I was not trained in the small animal area, I observed and took notes on my observations in the small animal room that housed smaller animals such as rabbits and birds. Volunteers or shelter workers did not regularly staff this room.

Participant observations were conducted during regular volunteer hours and ranged from 9:00 am to 7:00 pm. Most observations were conducted on the weekends, 
with 7.5 observation hours conducted on weekdays. Two classes were observed, TTouch for cats and Dog Safety and Handling as part of the new volunteer orientation.

\section{DATA ANALYSIS PROCEDURE}

Qualitative data analysis is primarily inductive, in that data are emergent (Lincoln \& Guba, 1985). In order to understand the gathered data, I used both etic and emic approaches to data analysis. An etic or "outsider's" approach is one that is a description by the observer (Peterson \& Pike, 2002). An emic, or "insider's" approach is an attempt to "portray the world of the site in terms of the constructions that respondents see" (Lincoln \& Guba, 1985, p. 365) that includes expressing informant's constructions in their own language.

Taking into account both etic and emic approaches to data is crucial within qualitative methodology, particularly ethnography, as I was participating in both perspectives. As a participant observer, I shared in the activities of the setting through volunteer work and participated in the educational workshops and therefore developed an insider's, or emic perspective. At the same time, I maintained an etic or outsider's perspective in order to maintain distance to report upon the setting (Patton, 2002). I was at the site as a novice volunteer and was a volunteer for under a year at the shelter and so was able to maintain an "outsider's" perspective on the shelter as well.

\section{Thematic Analysis}

Thematic analysis is often used within qualitative research because this data can provide first-person insight into individual's experiences, beliefs and motivations. An advantage to analyzing themes within ethnographic research in particular, is that the 
thematic data can be reported in a way that uses transcripts and field notes as cultural texts (Luborsky, 1994).

Thematic analysis is useful within qualitative research because themes are culturally situated, in that for many people, themes make up a part of experiences in daily life. However, investigators should take care in relying solely on thematic analysis of data due to the fact that all informants may not share personal themes. Luborsky cautions that themes derived from interview data should be examined critically, and should not be over generalized. Rather it should be understood that "themes are markers of processes, not fixed structures" (Luborsky, p. 192) and themes are both emergent and changeable, and may vary over the course of a lifetime in both desirability and meaning.

I examined three forms of collected interview data; individual interviews, fieldnotes and documents for themes and patterns. All interviews were transcribed and read several times.

In this first phase of analysis I counted and highlighted words and phrases that occurred most frequently or were repeated, looking for patterns. Second, I went back over the interview transcriptions, noting patterns of speech, words and descriptions of experiences and grouped these descriptions into similar categories to identify main points or topics. Within the transcriptions and field notes, I underlined talk that participants said with emphasis, or noted intensifiers within the transcription.

In this second phase of analysis, I also noted evaluative clauses which can highlight the speaker's reasoning, beliefs and judgment and are often "identified by the expression of moral language" (Lubrosky, 1994, p. 199) and words and phrases were highlighted that occurred frequently and appeared essential to the interview participants' 
experiences. After each transcript was read several times, I turned to my field notes and observation data to see if the same patterns emerged.

\section{Communicative Events}

Communicative events are an important component of data analysis within Ethnography of Communication. Analysis of a speech event, or communicative event is important so that similar observations conducted at different times can be compared and so that "generalizations can be made about patterns of communication within a constant context" (Saville-Troike, 2003, p. 25). Following the method of EOC, I recorded two speech events that occurred within the shelter in order to examine the key units of communication within the shelter.

\section{$\underline{\text { Data Management }}$}

Ethnographic data collection and analysis often occurs simultaneously, and accurate data management and retrieval is integral to qualitative ethnographic methods (Hammersley \& Atkinson, 1995). Therefore, keeping data organized is key to accurate analysis (Lofland, J., Snow, D., Anderson, L., Lofland, L., 2006). In order to keep my interview data organized I took notes either during my observations or immediately afterward. Paper printouts were organized and kept in a locked file and electronic data were stored in password protected electronic files. Both were organized according to date for accessible retrieval and revised coding throughout the data analysis process. 


\section{COMPARABILITY AND TRANSLATABILITY}

Ethnographic research occurs in natural and unique settings therefore researchers generally take the approach of comparability and translatability rather than aim for findings that transfer directly to a wider population, i.e. generalisability (LeCompte \& Goetz, 1982). Comparability calls for the researcher to "delineate the characteristics of the group studied or constructs generated so clearly that they can serve as a basis of comparison" with other groups (LeCompte \& Goetz, 1982). I kept a list of terms and definitions that I clarified with shelter workers in order to ensure that these constructs and definitions are accurate and could serve as a basis for comparison with other similar groups. This process is called member checking, in order to ensure that these constructs and definitions are accurate and could serve as a basis for comparison with other similar groups (Lincoln \& Guba, 1985).

Translatability presumes that the methods used and descriptions of groups and categories are explicitly defined so that comparisons can be conducted either by this investigation or other researchers. In order to ensure both translatability and comparability, this study specified how the research was designed, conducted and carried out, leaving an external audit trail (Lincoln \& Guba, 1986).

Although ethnographic research aims for translatability and comparability, problems of reliability and validity also play important roles within qualitative ethnographic research. ${ }^{2}$ Reliability is defined as the degree to which the findings are "independent of accidental circumstances of the research, and validity can be described

\footnotetext{
${ }^{2}$ Reliability and Validity are terms used in earlier ethnographic writings. Translatability and Comparability are more commonly used today.
} 
as the degree to which the findings are interpreted in a correct way" (Kirk \& Miller, (1986, p. 20).

\section{Reliability}

LeCompte \& Goetz (1982) state that external reliability in ethnographic research is dependent on five factors. The first, Researcher status position, or the social status the researcher may have can influence the information provided by informants. I entered the field as a new volunteer, therefore my "position" at OHS was that of novice. Although I entered as a graduate student, the age, education level and experience widely varied among my informants, and I could not confidently assume that my social status due to education level was higher than my fellow volunteers or staff, thereby influencing the information they provided.

The second is Informant choice, or the individuals chosen to participate in the study. I understood that associating with certain groups may have precluded access to others, and informants who may be able to provide detailed information may be atypical for their group. In order to select a range of interview participants, I chose individuals whose experience at the shelter ranged widely from two months to thirteen years and two participants were recruited though judgment sampling from different parts of the shelter.

Social situations and conditions are the social situations or circumstances that may influence the informant's participation. I was conscious of the context in which I gathered data, in that social situations may influence what informants reveal. I imagined that some interview participants might be uncomfortable talking about sensitive experiences at their place of work at OHS, and I wanted to ensure that they had a choice in whether to participate in the interview on, or off-site as noted earlier (pg. 22) as an 
alternative to meeting at the shelter. As a result, 6 of the 8 interviews were conducted away from $\mathrm{OHS}$.

Finally, as a further check of external reliability, methods of data collection and analysis are clearly defined and include the research design, data collection and analysis so that my process is transparent. I took detailed field notes so that they can be an additional check of reliability to specify the "context of observation" for the reader (Kirk \& Miller, 1986).

Internal reliability is concerned with the extent to which the meanings held by observers and participants are similar. This can be achieved through low-inference descriptors, or verbatim accounts of individual speakers including narratives of activities and behavior. In order to ensure that I was recording informants verbatim, I recorded all interviews and narratives using a digital recorder.

As I was the sole researcher at OHS, and therefore unable to confer with multiple researchers or use peer examination, I used member checks as a way to check if my interpretations match those of my informants (Lincoln \& Guba, 1985).

\section{Validity}

Validity is concerned with whether the theories generated or tested match with the actual conditions of the study (LeCompte \& Goetz, 1982). Internal validity was addressed by the following. The issues of maturation and mortality of a community can be looked at as topic of study in itself and can be used as data if the change is significant. However, as I was at the site as a researcher for 9 months, I did not witness a high level of community change. 
Observer effects can occur when informants become dependent on the researcher for enhancement of status or other psychological needs. I chose a diverse group of participants in age and length of time at the shelter in order to establish several different relationships at the site. In addition, I was aware that I was working within a social setting, and my presence may affect the setting (Saville-Troike, 2003). I attempted to be unobtrusive when taking notes on site and recorded detailed notes after observations so not to be distracting to the community I was participating in.

Spurious conclusions can be guarded against by eliminating rival explanations through maintaining efficient retrieval systems for data as well as use of "corroboratory and alternative sources of data" (Saville-Troike, 2003, p. 50) or triangulation of data, (Patton, 2002) two approaches I used in this study.

\section{Data Triangulation}

Triangulation of data is identified as an additional way for researchers to strengthen their research and guard against errors. By combining data from observations, interviews and document analysis the fieldworker is able to use different sources "to validate and crosscheck findings" (Patton, 2002). By taking care to bring evidence from several sources, and testing this data against one another, triangulation can increase validity.

External validity is concerned with identifying distinct characteristics of the population of study that can be compared to other similar groups (LeCompte \& Goetz, 1982). In order to ensure that both researchers and participants agree upon the meanings and interpretations of constructs (LeCompte \& Goetz, 1982), I asked the members for 
clarification and origin of terms to check the accuracy of constructs and researcher interpretations.

\section{Researcher Position}

Within qualitative inquiry, the researcher is used as a tool for information gathering and data analysis (Patton, 2002). Scholars state that qualitative research is not value free (Lincoln \& Guba, 1985); therefore, adequate self-reflection and disclosure by the researcher about the researcher's motivation, biases, and expectations are an integral part of the qualitative research process. I recorded statements of any biases and expectations about the study subject matter before and after completion of the study as well as during the data gathering stage (Appendix A). My biases and assumptions were disclosed in order to both to maintain awareness of my thoughts as a researcher, and reduce influence over my data collection and analysis.

Identification of how a researcher's values and ideologies may influence a study requires the investigator to be reflective (Creswell, 1998). I kept detailed notes on my reflections of my experiences before and during my time in the field. As a volunteer, I provided labor to the organization, therefore providing reciprocity. The idea of reciprocity is that the researcher should "give back" or contribute to the host group in some way as they are using individuals time (Fetterman, 1989). All informants remained confidential and are identified by pseudonym only in field notes. To further guard participants' identity, any direct quotes from interview participants are identified in the findings and discussion portion of this study as staff 1-3 and volunteers A-E.

The next chapter discusses research findings including Ethnography of Communication components and the overarching theme of relational bonding. 


\section{CHAPTER IV}

\section{FINDINGS}

\section{INTRODUCTION}

The purpose of this study was to understand the culture-sharing group of people working within the Oregon Humane Society, a Pacific Northwest animal shelter, and present the findings from eight individual interviews with Oregon Humane Society shelter staff and volunteers, 40 participant observation hours within the shelter and analysis of a set of organizational documents and artifacts. This chapter opens with a description of the demographic characteristics of the study participants followed by discussion of the Ethnography of Communication and concludes with an explication of the key overarching theme in the data of relational bonding.

\section{DEMOGRAPHIC CHARACTERISTICS}

The term "shelter workers" will be used within this section as well as subsequent sections in this chapter and in Chapter V to refer to both volunteers and staff where they are referenced to as one group; otherwise they are discussed separately and titled as such: volunteers and staff.

Criteria and Sample Size

Criteria for selecting volunteers and staff were those who worked primarily in the cattery or kennels with direct contact with animals for at least two months. I chose a wide range of experience (two months to 13 years) for interview participants in order obtain the perspectives of individuals who were presently learning the culture of the shelter as 
well as those who had lengthy experience with the community. At the time of data collection, total staff and volunteer population at the shelter were 1,458 . The number of male volunteers was 236 and female volunteers 1,1101 . The male staff total was 27 and female staff was 94 . As both male volunteers and staff comprised $18 \%$ of the total shelter population and therefore significantly less than the female population, the sample interview population for the study reflected this ratio.

\section{Animal Shelter Volunteers}

Interviewing volunteers was important to understand the culture of the shelter as volunteers comprise over $90 \%$ of the total animal care population at OHS. Shelter volunteers in this study included four females and one male whose experience ranged from five years to thirteen years. Their experience at the shelter ranged from direct animal care, showing animals to the public, mentoring new volunteers, leading volunteer groups and teaching classes. Three female and one male volunteer worked primarily with dogs, one female volunteer worked primarily with cats.

\section{Animal Shelter Staff}

Shelter staff in this study included two females and one male whose experience ranged from two months to four years. Their experience at the shelter ranged from showing animals to the public, public service coordinator and direct animal care. Two females worked primarily with cats, one volunteer worked primarily with dogs. As this study focused on the animal care portions of the shelter only, participant recruitment was limited to Animal Care Technicians (ACT). To protect confidentiality, direct quotes from interview participants are identified in this study as staff 1-3 and volunteers A-E. 


\section{$\underline{\text { Artifacts }}$}

I collected and analyzed documents including 10 volunteer emails, printed packet of handouts for new volunteers, two monthly magazines, two promotional mailers and photographs taken onsite by the researcher.

\section{Field Observation}

I began as a new volunteer and both observed and participated in shelter activities. As a volunteer, I spent a total of 40 hours of participant observation over 9 months in the shelter areas from July 2010 to March 2011. This included 30 hours in the dog shelter area and 10 hours in the cat shelter area. Participant observations were conducted during regular volunteer hours and ranged from 9:00 am to 7:00 pm. The majority of observation hours were conducted on the weekends, with a total of 7.5 observation hours conducted on weekdays.

\section{$\underline{\text { Notation system }}$}

Underlined words or phrases that appear as data excerpts in this study are those that were emphasized in tone or volume by interview participants, or appeared verbatim in printed communications. Breaks in speech recorded within interview transcriptions or in field notes is indicated with three periods (...). Speech that is cut off mid sentence is indicated as a dash between words (-).

Although each interview participant was initially identified by a pseudonym, I have assigned a notation system corresponding to each participant to further protect confidentially. Shelter staff interview participants are identified in this study as numbers 1-3 and shelter volunteers, letters A-E. I assumed that some shelter animals might be recognized by their name, thus linking their identity to shelter workers who might have 
worked with them. Therefore I have omitted some names in the following sections as well as the Discussion chapter. The following section begins with a discussion of the key features of the Ethnography of Communication.

\section{ETHNOGRAPHY OF COMMUNICATION}

Ethnography of Communication was used as a descriptive theoretical framework to guide this study of the speech community at the Oregon Humane Society. This study sought to understand the OHS speech community in the context of its uses, functions and patterns (Hymes, 1962). As part of understanding a community of speakers, a list of terms was recorded from observed shelter communication in order to reflect the culture of the community of speakers (Appendix E). Terms and their definitions help in understanding the symbolic system and ways of being of the speech community and those features that are salient to the members.

The following section will illustrate the community of speakers within the shelter and provide direct data excerpts to illustrate the forms of communication found in the shelter.

\section{OHS Community of Speakers}

Within EOC, the social unit of a community is termed the speech community and is the social unit of analysis (Gumpertz \& Hymes, 1972). The speech community is a social rather than purely linguistic term that includes all forms of language including writing as well as nonverbal communication (Saville-Troike, 2003). "The vocabulary of a language provides us with a catalogue of things considered important to the society, an index to the way speakers categorize experience ...The grammar may reveal the way 
time is segmented, beliefs about the animacy and relative power of beings and salient social categories in the culture" (Saville-Troike, 2003, p. 28).

The speech community examined within this study was the community of animal shelter workers at the Oregon Humane Society. As OHS is a large organization with different departments, this study focused on the animal shelter kennels, where shelter volunteers and staff had direct contact with animals in their work.

Common Knowledge \& Beliefs about Language Use

Common knowledge within a speech community can be described as assumptions such as "everybody knows" or "of course," and are often considered "facts" that are assumed by the community of speakers (Saville-Troike, 2003). Included in common knowledge is a shared belief about who or what may be communicated with (Saville-Troike, 2003). The following statements demonstrate shelter workers' assumptions about what are shared understandings including the relationship between companion animals and individuals.

Um, I know that everybody there talks about respecting the animals so... (Volunteer A)

So, um there is just a trust that I love to establish with a dog, and it's such a sense of accomplishment to be able to create a bond with an animal. And, of course you're going to do that in your own home with your own dog, your own cat. (Volunteer B)

But yeah, everyone I know who works there since they've started working there either considers adopting a special needs animal or they have adopted one. (Staff 3) 


\section{$\underline{\text { Shelter Communication Forms }}$}

Findings revealed three main communication forms within the shelter: verbal between shelter workers and animals, shelter workers and the public and shelter workers to shelter workers, written communication between shelter workers and shelter workers, shelter workers and the public, including print and electronic publications, nonverbal communication between shelter workers and shelter animals, and between shelter workers and shelter workers including images and diagrams.

\section{Verbal}

It was found that shelter workers verbally talked directly to shelter animals:

As I scratch ___s side, a young female staff member in a pink OHS T-shirt appears in front of the kennel and inserts a bright neon-pink card in a clear plastic sleeve attached to the front of the kennel. "Ok , hopefully those people will come and get you." (Field note, October 2010)

She [volunteer] lets herself in and leaves my lap to greet her. I see her reach down with a long green blade of grass and takes it to eat. "Here's some greenery." And then, "You have some noisy neighbors." (Field note, October 2010)

I heard the volunteer who had helped me talk to one of the dogs, , an older mix in the yellow pod. She spoke to her directly, asking how she was. (Field note, August 2010)

Verbally communicating directly with shelter animals appeared to a part of "common knowledge" or a shared belief about who or what may be communicated with (Saville-Troike, 2003). Such communication between shelter workers and shelter animals was acknowledged and encouraged within the shelter.

The more I realize that...the more I see how animals communicate with each other, I like to see how similar it is than when people talk with each other. Then I'm also intrigued by that we can actually do things with our hands and our bodies and our voices to communicate with them. (Staff 1) 
Within verbal communication, the naming practices of a speech community can be considered an important part of understanding a culture, as bestowing names often plays a part in altering the status or respect of those named in cultures (Saville-Troike, 2003). In the shelter environment, according personality through naming companion animals is a way to establish individuality and biography for the animal (Phillips, 1994, Taylor, 2007). Such behavior was evident at OHS:

Sometimes they don't know their name cause they just got one. (Volunteer C, Field- note September 2010) said that she was better at remembering the name of the dog rather than the kennel number. (Field note, July 2010)

The care that shelter workers took to properly identify animals by their correct gender was also observed:

She [dog shelter mentor] said she kept calling __ a "him" and checked her white paper collar to see if we were addressing her by the correct gender. This was confirmed. (Field note, July 2010)

Naming shelter animals is thought to provide an opportunity for workers to add meaning to their work, as well as "maintain the identities of the subjects in their care" (McKay-Semmler, 2007, p.15).

\section{Written}

Written or printed communication is considered codified speech and took several forms in this study. Written communication was found among shelter workers as well as printed and electronic publications.

Speaking for the animals through written messages composed by shelter workers to other shelter workers was common through white boards posted in both the cattery and the dog kennels: 
Tanya - 60 Needs a kennel mate, Sparky - 71 Separation anxiety/needs love, Stella -34 Go Slow! Very scared. Will growl or snap if pushed too far. (Field note, September 2010)

She said that if we take a cat out to show we should mark on the board with an " $\mathrm{X}$ " for that day so that if someone wants to see a cat, and the cat appears tired or irritable we can tell the customer that he/she needs a rest and to come back tomorrow. (Field note, October 2010)

Information about animals likes, dislikes and ideal home environment as indicated by either shelter workers or animals' previous owners were often written through the animal's point of view.

I notice that many of the outside cards I am reading are written in first person from the perspective of the cat, dog or rabbit/bird. I think about the purpose of this and challenge this norm by reading the cards differently, taking the "I" and replacing it with "He" and "She" and even "It." And it does sound odd, impersonal. (Field note, January 2011)

Signs observed on outside/public side of cattery cages that appear to note demeanor and preferences of each individual cat. "I'm stressed," "Please do not put fingers in here," "I'm feeling a little insecure." (Field note, January 2011)

Hi my name is Boss! You will find that I want to be as physically close to my human friends as possible. Cuddling is my favorite activity in the world! My friends here at OHS think the perfect home for me would be one with older children that can understand that I need to be handled gently. (OHS volunteer email, April 2011)

The printed information, or "kennel cards" displayed on the front of shelter animal's kennels for the public were often reproduced on the OHS website and included in volunteer emails as "featured animals" of the week. The kennel cards often included brief stories of the animal written by OHS staff.

Mrs. Sweet Pea is a real Lady. She is sweet and affectionate but will also let you know when she would like some time alone to reflect. (Volunteer email, February 2010) 
In a volunteer email that gave an update on an "OHS alumni" (shelter animal that had been recently had been adopted) more than half of the update was written in the voice of the dog:

This is a message from Buffy herself! Since I left the Oregon Humane Society, things have been going spectacular. So, let's recap a bit. I was not a happy girl in my kennel. Being blind, things were very scary sometimes. Lot's of noise that confused me... Ah, they have figured out I love to have my belly scratched and my neck rubbed...Daddy is already spoiling me. Hopefully Papa won't make him stop. What do you think of my new collar?...Yes, life is wonderful! (OHS volunteer email, October 2010)

Written communication was also found in the form of magazines, pamphlets and training materials for new volunteers, again often "speaking for" the animals:

We need your support to make the dream of a home for every cat and kitten come true. (OHS appeal pamphlet, 2010)

In the following excerpt from a letter printed in an issue of OHS magazine, an adopter explicitly mentions relationship building with a newly adopted shelter animal:

Today I took her for our first run and she is a perfect companion. She and I are still building our relationship, but feel very solid with our beginning. (OHS printed communication, 2010)

\section{Nonverbal}

Nonverbal communication was also a significant channel of communication within the shelter. Nonverbal is used to describe human communication that moves beyond written or spoken words (Knapp, 1980) and can include silence, kinesics, proxemics, eye behavior and images (Saville-Troike, 2003).

All category types of nonverbal behavior were noted within the shelter. Silence was noted within the shelter setting. Eye behavior in terms of avoiding eye contact with some animals to reduce the animal's fear, and images were also used within printed 
communication in the form of pamphlets, and content on both OHS website and magazines.

The nonverbal is an important communication channel that may provide information that sometimes cannot be obtained from verbal behavior (Ekman \& Friesen, 1974) and can be used to express our relationships with others as well as our feelings toward them (Trenholm \& Jensen, 2007). Nonverbal cues play an important role in "situations where verbal communication is constrained" (Knapp, 1980, p. 16). In the case of the humane society, nonverbal communication was relied upon when "meeting" or communicating with shelter animals.

As new volunteers, we were encouraged to learn the behavior of shelter animals. Animal behavior was taught formally in classes as well as encouraged within shelter documents. As new "dog walking" volunteers, we received a packet of information including a full-page "Visual Glossary" of drawings of dogs to help us identify seven different body positions from "Relaxed to "Offensive Threat" that we might encounter as we interacted with shelter dogs (OHS, 2010) (Appendix F). In this way, new volunteers appeared to be learning a particular way of interacting with shelter animals.

I think for us to try and be aware of what their [shelter animals] body language indicates and how they're communicating with each other and how they're communicating with us though this different language. It makes us have to be aware of that and then we also are maybe a little more alert to what people are communicating to us. (Staff 1)

But, I think that to develop a bond with a dog you have to be able to read the dog and listen to what the dog is telling you. And, they communicate really, really, really well without saying anything, you know? I mean once you're around dogs enough, you can definitely tell what that dog is saying... (Volunteer B) 
In a training packet for new volunteers, the importance of shelter workers own nonverbal communication or "body language" was stressed when interacting with shelter animals:

\section{Your Body Language}

- Remain calm and neutral:

- Avoid direct eye contact.

- Stand upright or bend at the knees.

- Stand at a slight angle instead of directly facing the dog

- Keep the dog in your peripheral vision

- Keep your hands low and relaxed. Fold your arms if a dog is grabbing at your hands

- Speak softly

(OHS, 2010)

In a dog safety and handling class, the instructor demonstrates for new volunteers

the experience of shelter workers "invading" the space of shelter animals:

She asks for three volunteers to help demonstrate that we should not crowd the dogs in the kennels when we enter or hover, asking the three people in our group to come up in the middle of our circle of chairs to "pretend you are in an elevator" and they stand close together. then enters their space and stares at the each one directly in the eyes, inches from their face. She says this would be socially awkward and "not polite" and that dogs can feel the same way in their kennels when we enter and look at them. She states that we should be aware of this when we enter, as dogs are social, and can become similarly uncomfortable. She states that the kennel may be unfamiliar to them and that they are used to being with people in a home environment and the shelter may be strange to them. (Field note, September 2010)

One staff member described part of his job as a translator of animals' nonverbal

language to humans.

Anyway that I can describe them and almost act as a, as a translator in many ways (laughter) for them... Because you know we have our behavior department puts on these classes about canine body language and they have their basic training classes. And when you take these classes you start to learn about something as simple as wow, that tail is up and wagging versus okay, it's tucked underneath the legs, what that means. What this dog's open mouth breathing is versus closed mouth and staring at that person. What does that mean? So, some people are not gonna be as quick to see that. And we've seen thousands of videos on oh look, 
here's a dog bite video and wow this dog didn't indicate that it was going to bite it just stood there and then it bit. But when you watch closely and slow down these videos you look at all these signals and so many people don't see the signals. So yeah, I guess in many ways we act as translators, we say no here's your signal, here's your signal, here's your signal, this is how you could have avoided getting bitten in the face so in many ways it is like translating. (Staff 1)

Touch is most likely the most basic form of communication (Knapp, 1980) and appears to be an important feature of shelter communication. Within the Tellington

Touch (TTouch) class taught at OHS, there was emphasis on the use of touch specifically to help make shelter animals more adoptable by releasing tension and fear:

You know, I love dogs and so just to be able to spend some time with a dog, take him out of his kennel, um interact with him and help him feel more comfortable, confident, relaxed um even if he just sleeps for a little bit too cause I imagine that they probably don't...some of them don't sleep a lot cause there's other dogs barking and it's a strange place and it's kind of scary and so just to take him away and do some touches and help them relax and even if- especially if they you know close their eyes and doze for a little while (Volunteer A).

Another goal of TTouch is building a bond with the animal you are working with:

We should remember to be present and focused with the animal, and that T-touch can deepen the bond that you have with the animal. "This is the biggest benefit." (Field note, November 2010)

One shelter staff member describes touch as facilitating socialization for cats in the cattery:

I really like when we get in cats that are scared or their previous owner said they weren't sociable, weren't a good companion animal and when they stay with us they sort of come out of their shells cause we have lots of volunteers that come in and work with them by just petting them gently and trying to get them to come out and be more social (Staff 3).

Silence was also a feature of the shelter. When I had first started volunteering, I had expected there to be more talking amongst staff and even to the animals. What I discovered was that the shelter workers who appeared the most experienced talked less 
and appeared to rely primarily on non-verbal communication when taking dogs out to walk.

The volunteers appear to usually be very busy doing their jobs walking dogs and do not necessarily socialize with one another. (Field Note, September 2010)

We introduced each other, but were fairly silent on the walk together, each of us concentrating on our dogs. (Field note, August 2010)

As we were to rely on many nonverbal cues to "read" an animal when taking them out of their kennel or interacting with them, silence appeared to assist in the process of building trust with an animal, as we were discouraged from using "high-pitched or excited talk" (OHS, 2010). Silence is often regarded as the absence of speech or communication. However, silence can act as part of communicative interaction, and act as a kind of "speech" act in itself (Saville-Troike, 2003).

\section{Shelter Space}

In Ethnography of Communication, the organization of space is an important component of analysis because the way individuals interact within a particular space can be considered nonverbal communicative behavior (Saville-Troike, 2003). Discourse surrounding shelter space was observed within OHS:

Shelter animals that are "on the floor" or ready for adoption are called the "cattery" for cats and the "kennels" for dogs. From the main shelter entrance, the dog kennels are entered through the right side of the lobby and the cattery on the left. In order to reduce the stress of animals from encountering one another, and to facilitate interactions with the public, both the dog and cat kennels are public facing and no kennels face each other (Oregon Humane Society, 2000). 
The cattery area is shaped in a "U" with cat kennels ringing the inside perimeter. A portion of the wall that divides the cattery from the lobby consists of a glassed in area where visitors can view cats in a room with play structures and toys. There is an inner volunteer and staff room with two doors at either end. Records on cats are kept here, as well as cleaning supplies. Cats are taken out and put in kennels within this inner staff/volunteer space. Cats are occasionally taken out of their kennels and allowed to roam within this area. When this occurs, paper "loose cat" signs are hung on both doors to warn cattery staff that enter and exit this room. Due to the fact that the cattery space is smaller, cattery shelter workers and the public are physically closer and appear to interact with each other more directly and frequently than within the dog kennels.

It is very, very busy today [in the cattery]- visitors and staff are having a hard time maneuvering around each other in the space. (Field note, November 2010)

The Dog pods are physically a larger space than the cattery. The kennels are laid out kind of like a foursquare, with each "pod" color-coded and a schedule of which pod to go out first on each day was posted. We are to access the dogs via the gated corroders between the $U$ shapes of the kennels, behind the view of the public. (Field note, July 2010)

Shelter staff appeared to take care that a cat was in view of customers walking through the cattery:

A customer comes up to me as we are looking in at the outside of a kennel. The cat does not appear to be inside. She asks me if he is away and I tell her I will go check. I see an ACT in a brown OHS T-shirt and I tell her someone is looking at the cat and wondering where he is. We both peer in seeing that the cat is in the kennel, simply hiding inside a small cubby with a blanket thrown over the top. She adjusts it a little by pulling it forward and exposing a little to the outside "public" side. (Field note, February 2011)

Interacting with dogs in their kennels served another purpose besides purely socialization. The scene that was created for the public walking by the kennel of a person 
interacting with a shelter animal "modeled" the way the shelter animal might act with that potential adopter:

This creates a favorable impression for the public, as they may walk by and see a dog interacting with someone they then can see that scene in their home, thus potentially making that dog adoptable. This also may approximate an environment that the dog may have experienced in their former life, of hanging out with a person in a home-like environment. (Field note, September 2010)

To end the session, I found a large, older German Sheppard Bennie, and sat down with him in his kennel. As I was sitting with him, a man and woman walked by and said they didn't realize someone was there. They looked at us and remarked on Bennie. (Field note, August 2010)

The importance of making sure a dog was on display for the public (as to not miss

being be adopted) was noted by one shelter volunteer:

Y'know everything's a compromise. Whether you clean a kennel, um that's clean enough to eat off the floor, or whether you go in and clean up the pee and the poop and move on. Because you could clean it up so that anyone would want to eat in there but that's three or four hours the dogs not seen by the public. So, you have to compromise and say I gotta do just a quick clean so the dog can be seen as people come through and do that. (Volunteer D)

\section{COMMUNICATIVE EVENTS}

In addition to the foregoing, Ethnography of Communication calls for analysis of key communication components within a community of speakers: Speech situation, speech event and speech act (Hymes, 1974). By analyzing these components, the researcher is provided a base with which to analyze the individual units, as well as a way to understand how the separate components relate to one another. Through an analysis of communication events, the goal is to understand a speech communities social organization and salient features of the culture. 
The description of the components of the event generally include the topic, purpose or function, setting, key (intonation), participants, message form (vocal/nonvocal), content, rules for interaction and norms for interpretation.

The speech situation is the context within which communication takes place and a speech event is an activity that is directed by rules or norms for speech use. A speech act includes an action with a single interactional function, and the meaning often depends on the context of the speech community.

Two speech events were selected for detailed analysis. The first, a class for volunteers and second, a member of the public "meeting" a shelter animal. Both events were selected for frequency of occurrence and are examples of the importance placed on relational bonding with shelter animals. 


\section{Speech Event I}

T-Touch for Cats

(1) TTouch instructor/OHS volunteer holds a class to teach a group of volunteers about Tellington Touch (TTouch) a method of touch that shelter workers utilize to calm animals and reduce their stress.

TOPIC: OHS volunteer and certified TTouch Practitioner teaches TTouch to OHS volunteers.

FUNCTION/PURPOSE: Class to teach OHS volunteers about TTouch and how to use it. SETTING: In the Animal Medical Learning Center, Manners Hall 1 in OHS. A medium sized room with one window at the far end. Folding chairs ring the room with a large table at the back. There are photos on the wall and a mirror hanging on the wall at end of the room.

KEY: Formal, with intermittent humor from P1.

PARTICIPANTS: P1: TTouch instructor, P2: 8 Volunteers.

MESSAGE FORM: Spoken English P1 speaks to instruct, P2 offer comments and questions.

MESSAGE CONTENT: Informing volunteers about TTouch and instructing them how and when to use it with shelter animals.

ACT SEQUENCE:

P1 Introduces herself and purpose of class.

P1 Passes around printed material on subject matter.

P1 Demonstrates a series of touches in the air, P2 mimics her.

P1 Demonstrates on a stuffed animal. 
P2 Volunteers practice touches on each other.

P2 A volunteer goes to the cattery and brings back a cat to be a model for the class.

P1 Talks to cat and demonstrates touches on the cat and asks what we observe.

P2 Comments that the cat appears more relaxed.

P1 Dismisses volunteers to go to cattery to practice on cats.

\section{RULES FOR INTERACTION:}

P1 instructs and explains; P2 raises hand to request to speak or ask a question.

P1 Uses direct speech while teaching.

P2 Does not interrupt speaker while talking.

\section{NORMS OF INTERPRETATION}

TTouch is meant to reduce stress experienced by an animal. According to the trainer, TTouch "works with the nervous system to reduce stress" and in the case of shelter animals, to make them more adoptable because it releases tension and fear. It appeared that TTouch related to the overarching theme of bonding as the trainer stated as volunteers, we should remember to be present and focused with the animal, and that Ttouch can deepen the bond that you have with the animal. "This is the biggest benefit."

By reducing shelter animal's stress through touch, a shelter worker may be able to ultimately make an animal more adoptable, thus encouraging a relationship between an animal and potential adopter. The goal of making shelter animals more adoptable is echoed in the following narrative:

It just felt really um, rewarding to be able to go and help the animals to be more comfortable there so that they showed better. And people would take a look at them and say "Yeah, come home with me." (Volunteer A) 


\section{Speech Event II}

A member of the public "meets" a cat.

(1) OHS Volunteer shows a cat to a potential adopter from the public.

TOPIC: "meeting" a cat in a Get Acquainted room within the cattery.

FUNCTION/PURPOSE: Member of the public expresses interest to a volunteer about a cat that she has noticed within the cattery and indicates she would like to see the cat. SETTING: In the OHS cattery, a room situated near the front lobby. The cattery itself consists of an outer walkway that runs the circumference of the building, with an inner core or area reserved for the volunteers and staff. The inner room has two exits, one on either end of the room. In the room there are two computers and sinks, food, litter cleaning supplies, toys and beds. The wire cat kennels are ringed around this room so the volunteers and staff have access to the kennel backs to remove and place cats in.

KEY: Casual.

PARTICIPANTS:

P1: OHS Volunteer.

P2: Female Customer.

P3 Animal Care Technician (ACT).

MESSAGE FORM:

Spoken English P1 speaks to instruct, P2 offer comments and questions. MESSAGE CONTENT:

The cat, potential adoption and suitability of the home environment for the cat. ACT SEQUENCE:

P2 Verbally asks P1 if she can see a cat. 
P1 Agrees, brings out printed material called a kennel card about cat. The card generally includes a "recommendation section" which includes phrases such as "good with kids" or if the cat should go to a home as an only cat.

P1 Reads aloud printed kennel card which contains information adoption and medical staff have written about cat to P2.

P2 Verbally agrees to see the cat.

P1 Finds empty Get Acquainted room near the back of the cattery for P2 and cat.

P1 Brings out cat from kennel and places in Get Acquainted room with P2.

P2 Holds cat on her lap, strokes cat and verbally talks to cat.

P3 Enters the Get Acquainted room with P2 and they discuss adoption of cat. P3 asks questions about home environment including if $\mathrm{P} 2$ has other cats at home.

P2 Answers P3's questions about home environment and shelter cat.

P3 Expresses her opinion that the cat would be better suited to another home, as P2 already has a number of other cats at home.

P2 Decides not to take cat.

P1 Accepts cat handed to her and carries the cat back to the kennel.

P2 Exits the Get Acquainted room and continues to look at cats in the cattery.

\section{RULES FOR INTERACTION:}

P2 Asks questions and provides answers.

P3 explains preferences for home environment

P2 Verbally talks to cat 


\section{NORMS OF INTERPRETATION}

This speech event is significant as one of the goals of the shelter is successful adoption or "match" and this is an example of a speech event that occurs daily in both the cattery and dog shelter areas. The discussion that takes place with members of the public and shelter staff surrounding the adoption of an animal is one that does not appear to be taken lightly by the shelter. For example, on the shelter's website, the following passage explicitly describes the purpose of the Get Acquainted rooms: "Knowing you'll want to spend time getting to know a dog before making the life-long commitment, OHS has several indoor rooms designed to let you play as well as cuddle" (Oregon Humane Society, 2008).

In order to make sure adopters are matched with ideal homes, "meeting" and "getting to know" an animal does not appear to be a rushed process, as one of the goals of shelter workers is avoiding animals returned. Shelter workers appeared to encourage adoption if they determined the person was a good fit for the animal and the animal a good fit for the person. However, they appeared to actively discourage adoptions if they felt that it was not a good match:

More often than not, you're talking someone out of the wrong dog. (Volunteer C)

\section{RELATIONAL BONDING}

In looking at the shelter through an analysis of animal shelter communication, the findings of this study reveal a significant level of relationship communication within the animal shelter portions of OHS. Data analysis revealed an overarching key theme of relational bonding, which serves as organizing frame for data from interviews, 
observations and artifacts. Data presented within this section will include direct excerpts that reflect relationship-building processes directed toward relational bonding and it's opposite, relationship termination.

The following discussion is organized using set of terms which reflect the bonding theme, processes of bonding and categories of regularly occurring relational types within which bonding (or lack of) was a key element and central issue. Organized under the key, overarching theme of "bonding" this section will expand upon the findings which include a discussion of the theme of relational bonding occurring within an animal-centric organization, the themes of relationship communication and relationship network.

\section{$\underline{\text { Animal-Centric Culture }}$}

A network of relationship terms, types and processes found within the shelter have been categorized into four groups: A. Shelter animals and shelter animals B. Shelter workers and shelter animals C. Shelter workers and shelter workers D. Shelter workers and the public. Shelter workers acknowledged the existence and importance of bonds occurring between shelter animals. They also appeared to make efforts to bond with shelter animals, and shelter workers formed relationships with each other in part due to these efforts. Data also reflects shelter staff promoting public-animal bonding.

The relationship terms, types and processes discovered appeared to support an animal-centric culture within the shelter and the efforts of shelter staff flowed upwards in support of this animal-centric culture. Much of the language observed appeared to support an atmosphere in which animals were at the center of the worker's goals and efforts and promoted a public-animal connection: 
Um, the staff all seem to be really dedicated you know? It is for the animals you know? It's all about the animals. (Volunteer E)

She states that "The bottom line is we are here for the animals." (Field note, 2010, Dog Safety and Handling class)

And um, when you get there you are all there for the dogs and I think that's really something that is kind of a uniform saying amongst the people I know is that it's about the dogs. You know, it's not about us; it's not about the rules and all of that. It really is ultimately about the dogs. (Volunteer B)

The presence of the following repeated relationship building terms that support the overarching theme of relational bonding suggest that a network of interrelated relationships occurred in the service of bonding between: A. Shelter animals and shelter animals B. Shelter workers and shelter animals C. Shelter workers and shelter workers D. Shelter workers and the public. The following data excerpts illustrate the relationship language, process and relational types observed within the shelter setting. The set of relationship building terms include meeting/getting to know, socializing, spending time/visiting, trust and matching.

Bond

In what appears to be an animal-centric culture, I begin with a discussion of animal-animal connections. I first observed the word bond in initial observations of kennel paperwork. Two animals housed in a kennel together were marked in their paperwork as a Bonded Pair, meaning they had lived together in a previous home or became attached to each other at the shelter. Bonded pairs were only adopted out together in order to preserve the relationship and reduce stress on the animals.

Um, when a- to me when a cat is bonded to another cat, that means they need to go home together, they need to be adopted together, that we feel like that if they are separated there would be a detriment to their mental health. And that they would help each other get adjusted and that they would really do best as long as they stick together. (Staff 3) 
But I just look at that as... somebody's security, you know? That's your constant comfort and you don't want to... see them apart cause they just crumble when they're not together. (Volunteer C)

As a relationship term, bond was described as an "attachment" or "connection" that can form between animal and human.

In my line of work I guess the definition for bond would be a connection between two beings whether it be between two people, or a person and an animal, or two animals. And it would have to be a strong connection I think. (Staff 2)

I guess as a person, a bond you feel towards the animals that you are helping, or a specific one, you know, that you feel close or a connection to the animal. (Staff 3)

There have been a couple of dogs that I worked with when I was actively in the pet pals program that I developed a bond with, and I would go home and tell my husband "If we didn't already have a dog I'd bring this one home." Um but I knew there was- they were waiting for somebody else but I could still, um I could still open my heart to them, I could still let them in, I could still love them and appreciate their- the relationship that they had with me. I mean when I would go in there they would recognize me, they would wag their tails er, um smile, I believe dogs smile so um, so yeah. (Volunteer A)

One volunteer described a bond with an animal as something more than simply owning an animal:

And I consider myself very fortunate because I have friends who have cats and they're just cats. They happen to be pets that are in the household but there doesn't seem to be that bond. And I think if you take-if your lucky and you take the time to try and establish something with the animal, that's really special. (Volunteer E)

Other volunteers described encouraging the bonding process between shelter animals and workers in order to facilitate a similar relationship between the adopted animal and an adopter:

Well, I can see how that would be a word that would come up in this place because we're really interested in the bond that forms between the companion animal and the owner. We're in the business of companion animals, not barn cats and not you know, ratters, mousers, things like that. So, the adoption counselors 
are vigilant in screening the applicants, and they really care, they want to find good homes for the animals where the cats can form that bond with their owner or guardian. (Volunteer E)

Another shelter staff member described the ability that some shelter workers had

in forming bonds with animals at the shelter:

Um, we had a gal who just recently left OHS who was really good at forming bonds with small dogs that were sometimes aggressive. So she would just be able to get in there and talk to them and ease them down and by the end of the day she would be, like have them in her lap and they'd be like totally at home. But anyone else tries to go in there and they'd be like "Grrr" and baring their teeth. (Staff 2)

So, um there is just a trust that I love to establish with a dog, and it such a sense of accomplishment to be able to create a bond with an animal. And, of course you're going to do that in your own home with your own dog, your own cat. Um here, it's trickier and it's a challenge, and sometimes there not long enough here to create that but I love that little dance that you do at first with a dog, to let them read you and you read them um, the difference between a shy dog and a really pushy dog um, so there is a challenge to it, and I am a student. (Volunteer B)

On the other hand, another shelter staff member mentioned that she avoided

personally forming bonds with the animals she worked with at the shelter:

Well, personally I try not to form a bond with the animals that I'm showing. Uh because um, then I would be taking home all of the cats. And I now have one cat and she is very happy being queen. She doesn't want any other cats. (Volunteer E)

One volunteer described his interpretation of the process of bonding between a

volunteer and shelter dog.

There's a certain look that a dog has when they look at you from the perspective of "what are you going to do to me, I don't like you, I don't trust you." And then after a while you go through a phase where they look at you all the time cause they don't want-you're going to be staring at them, so you don't look at them, but they're staring at you cause their like whatta you doing. Then there's a time when they're not looking at you, and they're getting to know you and then there's a final time like maybe they're in your lap or their jumping up, or their doin' things where they have a totally different look and you go there's the bond. And you can see it in their eyes, I trust you. Um, For example a dog'll lay down next to you, put his back up against you. Well, he's no longer looking at you, it's a very vulnerable position, but he knows that you got his back. Literally, you got his 
back covered, if something' comes this way your going to take care of it, or alert him. So when-when I'm showin' dogs to clients and their sitting in there and finally the dog just, overwhelmed, or whatever the case is comes down and just bonk, back up against them that's a good sign. The dog trusts you. And that's part of the bond. (Volunteer D)

In describing the bonding process, the shelter volunteer draws on nonverbal cues from the animal he is interacting with such as "looking"/"not looking," "in your lap," “jumping up," or lying "with his back up against you." He describes the shift from nontrust to trust as a final component. The following sections describe repeated relationship terms that were discovered within the shelter that appeared to support the idea and goal of bonding:

\section{Meeting/Getting to know}

As shelter volunteers, "meeting" and "getting to know" an animal were stressed in formal classes, as well as emphasized by interview participants as a part of their everyday work.

I really like it, I like to know a lot of the dogs so I'll walk though the pods often times, even if I only have 20 minutes I'll walk though with treats I'll meet some dogs, I'll look a little bit at their profile and just see if there's a dog I really want to work with. (Volunteer B)

She says that when walking dogs, we should take the time to get to meet and "get to know" the dogs when we walk them. (Field note, Dog Safety and Handling Class September 2010)

Spending time with an animal was encouraged as part of our work as volunteers,

as well as an activity that many volunteers engaged in:

You know, I love dogs and so just to be able to spend some time with a dog, take him out of his kennel, um interact with him and help him feel more comfortable, confident, relaxed um even if he just sleeps for a little bit too cause I imagine that they probably don't...some of them don't sleep a lot cause there's other dogs barking and it's a strange place and it's kind of scary. (Volunteer A) 
Then I had to do some- I had to go be a Pet Pal do that training so I could really get some relationships going with dogs and spend extended time with them.

(Volunteer B)

"Meeting" an animal was used in the context of both understanding the

individual traits of an animal, as well as a process that was encouraged by the shelter if a customer appeared interested in an animal. The shelter had several rooms in both the cat and dog areas specifically for the purpose of providing a quiet place for a potential adopter and an animal to spend time together. The rooms were referred to as the Get Acquainted and Meet and Greet rooms.

A customer is looking at a cat in the colony room next to us and asks, "Would you like to meet her? If you want you can come into the room and talk to her to see what she's like." (Field note, October 2010)

And then I usually just hang around the outside of the cattery, um waiting for customers that need help, if they have questions or they want to meet a cat. (Volunteer E)

We've got dogs that have lived outdoors, if you'd like to meet one of those, let's go back, I'll help you find one. (Volunteer D)

\section{Socializing}

Shelter workers were encouraged to also engage with shelter animals in the shelter spaces. For example, volunteers often "socialized" with cats in the colony rooms within the cattery and played with dogs in the outside yards. These behaviors helped to "socialize" shelter animals to make them more adoptable.

So then you try to socialize them, and work with them and earn their trust. (Volunteer C)

Because most of the cats, especially the kittens get a lot of handling, they get a lot of attention...the exception might be the cats in the colony rooms because they don't have the- the people poking their hands through the cages trying to pet them and talking to them. They are behind glass and so they don't get as much 
interaction. So I encourage my new volunteers to um, to go in and sit down and interact with all the cats. (Volunteer E)

\section{Spending time/Visiting}

Members of the public were encouraged to "spend time" and "visit" with shelter animal prior to adoption, or if they were considering adoption. If a potential adopter had another animal at home, they were encouraged to arrange a "pet meet" between both animals in the Get Acquainted and Meet and Greet rooms.

You may use cattery rooms for a rabbit shown to a client if they want to spend time with it on the floor... (Volunteer email, 2011)

You know, not everybody gets really attached to certain dogs because they didn't have the opportunity to really spend time with them. (Volunteer C)

Trust

Trust or building trust was also a term discussed within the shelter in relation to shelter workers and animals, often within the context of building a bond:

So, um there is just a trust that I love to establish with a dog and it such a sense of accomplishment to be able to create a bond with an animal. (Volunteer B)

Uh, a cat that's maybe shy that needs time to earn your trust. (Staff 4)

Trust was also mentioned as a specific goal of shelter workers within OHS

training literature:

\section{Establishing trust with dogs}

- Approach animals with confidence as well as caution

- Crouch at animal's level

- Avoid leaning over animal

- Move slowly, reach out with palm down, allow dog to smell your hand

- Maintain safe visual contact, but avoid direct eye contact

- Speak with soothing tones, avoid high pitched or excited talk

(OHS, 2010) 


\section{Matching}

"Matching" "making matches" or "match" was used to describe the process of using information shelter workers learned about members of the public and information about shelter animals temperaments, personality and backgrounds to create an ideal fit for both the animal and potential adopters.

I tell people I'm a matchmaker. I introduce dogs to people and people to dogs. That's what I do. (Volunteer D)

So matching to me is fitting. Or trying to set something up for what may be the perfect situation, or as close to the perfect situation as possible when it comes to different backgrounds. You know we have people come in looking to adopt and they work long hours so we're going to match them up with an animal that's maybe a little more independent, that's not gonna show separation anxiety when they're gone. Maybe an animal that has experience in a home, that knows how to be housebroken, we're going to look for ways that each animal that can fit into this person's lifestyle and at the same time, we're looking for a person who can accommodate sometimes these animals have needs that need to be met, meds, chronic issues. (Volunteer 1)

I can imagine a blind diabetic dog would be hard to place. Um, but again it was somebody that had experience with a dog like that before so it just sounded like it was a match made in heaven. (Volunteer B)

I [the animal] love to cuddle and be close to people so if you like love and affection too, we'll be a perfect match. (OHS Printed communication, Animal Description, 2011)

You know the immense significance of making sure that any dog you are showing to the public is the perfect match because the last thing that we want to see are any dogs being returned back to the shelter. (OHS Printed communication, class description, 2011)

The ability to "read" potential adopters in order to make good match appeared to be a skill that many shelter workers employed. For shelter workers, knowledge about individual animals appeared to help workers recognize when a member of the public was 
a good fit for an animal. The following narrative describes the ability to "read" a

customer in order to place a dog in a suitable home:

I get a feel for the people. (Volunteer D)

I try not to keep too much jargon to it, I just try and maybe sometimes my wording gears more toward their- what I've seen out of their personality and then I translate it over to more human characteristics when I'm describing them. You know oh, that's a weirdo, of he's a funny guy, or what a- what a lovely girl she is. Any, anyway that I can describe them and almost act as a, as a translator in many ways (laughter) for them is usually how I end up describing it to family and friends but mostly to the possible adopters is where you really see that come into play. (Staff 2)

And, y-you have to be able to look at the dogs and the people and you know, say this is going to work or this isn't going to work. (Volunteer D)

\section{Ideal Home or Adopter}

Matching was done with the anticipation that there was an ideal home or adopter

for a particular animal. Several shelter workers described finding the "right" relationship, or home for an animal.

And it was a perfect home, the people had experience with a diabetic dog before, and um, a blind dog and so it was just perfect. (Volunteer 1)

Well, I always consider that the most important part of the job is the customer service, helping people find the right cat. (Volunteer E)

Or, the one you match up and you say "Ooo- this is perfect, take this dog, this is your dog." (Volunteer D)

Um, their promotion is all about getting them adopted into the perfect home for them. So we work really hard trying to write their profiles, make sure that if the cat isn't going to be good with children, that we say that so that they don't get adopted out to a big family and then they're returned, we wanna make sure their first time, they get their forever home. (Staff 3 )

One volunteer described the satisfaction they felt of an animal going to a good

home: 
He was a red-reddish colored German Shepherd. Ba-rah-rah-rah. Was out in the kennels, out on the floor, running up, barking at everybody... So, made friends with him, worked with him, worked with him, worked with him. A week later, some people came back by, a couple came back by and __ was up at the front of the kennel wagging his tail and they said is this the same dog that was here last weekend? And I said yeah, that's __ he was right here. Last weekend he did this, and I said yeah, yeah you know it's not only been me but other people been working with him, trying to get him calmed down, and they said I wanna meet him. They met him and adopted him. And you know, somebody says well, how can you volunteer there? Well, that's how. (Volunteer D)

Other shelter workers described matches that were less than ideal:

That's the thing, cause you're better off keeping the dog and puttin' him in the right place than sending him out because people have the feeling that you're obligated you know? Like, this very wealthy looking couple came in on a Sunday afternoon and they wanted to see this little dog. And this little dog-up and down the fence, barking at another dog. I said, I don't know about you folks, but that would drive me crazy...I said, no, you don't want -- this is not your dog, forget it! ... And you could just see the relief on the guy's face, like I don't know how to tell you no. And I'm like you don't have to tell me no, I'm gonna give you the reason right here. You know? And they went yep. (Volunteer D)

The following narrative describes an adoption that appeared initially to be ideal,

and the frustration of a shelter volunteer when she learned the outcome of the adoption:

She went home with two guys that were supposedly the perfect home... When I called I said "How's doing?"... He said she tore up our basement a little bit so we had her somewhere where she- we opened the door to bring groceries in and she got away from us, and she was missing for like seven days on the street and then Multnomah County picked her up and they gave her an assessment and she didn't pass it and they put her down... But those people never called, never said "Our dog got away, should we do something?" And then, he said "I guess it was for the best." And you know, you go- you idiot. This was a rescue dog that was so shy. The reason she didn't pass the test was because she's a shy little dog. (Volunteer B)

One shelter worker described frustration with the public when they were advised against adopting an animal:

You hardly ever hear anyone say anything bad about an animal, but they will say something about the person. So there's-there's really a hard line that says pretty much the animals don't always do-they're, they're naughty. Or they're being a 
bad boy, or they're being a bad girl that kind of thing. But when it comes to people it's very direct. Stupid people. This person is crazy, let's get the dog. And we had to do that last week, a person adopted a dog, I told her not to do it, I told her she was going to have trouble, she did, she brought the dog back within hours...And so there's very much a feeling of-a open feeling if need be of contempt, when people are jerks and don't listen, and do that, we'll say stupid people. (Volunteer D)

Other shelter workers described disappointment if their perception of an animal

and the public perception of an animal differed. Below, a staff member describes the adoption of a cat she had grown "attached" to for the first time at the shelter that was later adopted:

And there was a couple that came in and visited with him and they wanted to adopt him, and they came up to the front desk and they're like, we want to adopt . And I was like "Oh my god, I'm so excited, he's my favorite cat; I love him." And I got no uh, feed-like emotion from them, they're like "Oh yeah." And so like, being so attached to this animal, I was like "Um, if you don't love him as much as I love him then I don't want him to go to your house" but you can't say that (laughter)... all I can do is look over the application, make sure it's filled out, make sure there's nothing suspicious about it, that he's going to be cared for, be inside, you know all that stuff. So I can't be like "No you can't have this cat because you don't feel the same way about him as I do." Um, so, you know that was kind of a let down seeing him go to someone who didn't seem as excited as I was about the situation. (Staff 2)

Volunteer $\mathrm{C}$ describes a similar frustration was when a favorite animal was not

viewed by the public in the same way as staff.

I mean, nine times out of ten I wouldn't tell them what they what we were going to see. I would just say "I know ever dog in the shelter, hands down, this is the best, best dog in the shelter. And has been the best dog for months of any dog that has come into the shelter." And they'd be like Oh, and then I'd be like asking them their situation and they' $\mathrm{d}$ tell me and I'd thinking like this is perfect. Walk around the corner, "This is _.." "Oh she's a Pit Bull, we would never adopt a Pit Bull." And I'm like you fool, do you say that about Black people too? I mean, my god you're so racist. If you aren't going to listen to any one here, and you know nothing about anybody here, and I'm telling you? So it was really frustrating, and I'd end up-they'd leave and I'd lay in the kennel with her for like an hour and just take a break, you'd have to like decompress. (Volunteer C) 
Matching appeared to be a skill that shelter workers employed not only to find a suitable home for an animal, but also to prevent the animal from being returned to the shelter. As can be seen above, this sometimes meant talking though the reasons a particular animal might be the wrong fit for a customer.

\section{Relationship network}

\section{$\underline{\text { Shelter Animals and Shelter animals: }}$}

I begin with what appears to be an animal-centric culture with a discussion of animal-animal connections (see page 53). As described earlier, "bonded pairs" referred to two animals housed in a kennel together that may have became attached to each other at the shelter or had been relinquished by the same previous owner. Bonded pairs were only adopted out together in order to preserve the relationship and reduce stress on the animals.

In order to avoid issues or conflict between animals that might be unfamiliar with each other, care was taken to avoid animals meeting "nose to nose" (OHS, 2010) in the hallways with the public or while out on walks with dogs. Volunteer training included specific routes to follow when taking dogs out, and remaining vigilant in "checking" hallways and when entering kennel rooms. This appeared to be for the safety of shelter workers, animals and the public as well as reducing conflict and stress on animals. Shelter space was designed in such a way to avoid animals directly facing each other in kennels, also in order to reduce conflict and stress on unfamiliar animals (OHS, 2000).

Allowing a customer's current animal to "get to know" a potential adopter's animal, a "pet meet" was often arranged between a potential adopter's current animals and a shelter animal. This meeting appeared to be an extra layer in the adoption process 
that ensured the animal would be successfully integrated into the new home, and also to avoid a return to the shelter:

Please bring your entire family to the shelter to meet your new pet and make sure everyone gets along well. That includes not only children (some pets don't do well with young children) but your canine family members as well. OHS can host "pet meets" at the shelter. (OHS, 2008)

In the following narrative, a shelter volunteer describes a conversation he had

with an adopter who was reluctant to bring their current dog into the shelter to meet a potential adoptee:

Do you have another dog? "Well we don't need to bring our dog in" (singsong voice). Yes you do. Cause I will tell you I've met this dog with other dogs, the dog wants to hump everything. "Well, my dogs not going to put up with that." That's right. Then we need to see when your dog tells this dog off. "Oh yeah, hadn't thought about that." Yeah your dog might not want to be mounted, but this guy ain't gonna stop. So let's see if he can be told to stop and respect that, or if he's just gonna be-you don't want a six-year-old dog that's being pestered by a two year old dog that wants to mount the dog every five seconds. That's no fair to the dog, any dog or for you. And...so, just don't do it. (Volunteer D)

\section{Shelter Workers \& Shelter animals}

Several narratives described the relationships between shelter animals and staff.

Several workers had animals at home that they had adopted from the shelter. Others had fostered the animals first, which meant they took the animal home with them while the animal was waiting to be adopted out. Sometimes workers grew attached to the animal and decided to adopt them. This was termed by one volunteer as a "failed foster."

You foster an animal and then you know, they live in your home, you get really attached and then sometime you can't let them go and then you adopt them back. (Staff 3)

So um, we fostered her and I adopted her and um, I mean, I guess bond? Like I never loved anybody, human or animal more than her...ever. Nor will I. I just know, I mean there's just like no way. So, she ended up dying of a weird 
complication from a cancer that I didn't even know she had. And she was gone, as soon as I noticed she was ill five days later she was gone. (Volunteer C)

And then my second cat was also a kitten and at the time I always thought I would just keep bringing the adults and the senior animals home, but kitten number two I was just very attached to, uh was trying to be adopted on our floor and then got really, really sick. He was scheduled to be euthanized, so I took him home had to force fluids in him cause he was just dying from what started as a typical upper respiratory infection and just engulfed him. So I had to force fluids, and give him meds. Finally he got better and after all of that time I just said I've invested too much to just let you go back to anybody. (Staff 1)

Other shelter workers described the adoption of an animal that had made an

impression on them as a kind of loss:

You know your heart always, you get a little choked up seeing some of them leave sometimes. And that was one for me. It's like all right, I'm waving goodbye, I know your going off well but boy, I think I have tears curling up in my eyes so I better just walk away now (laughter). (Staff 1)

I remember there was this one dog, and I can't remember his name now but he was a shepherd mix and um, I just really connected with him and we used to have what we called the chip yard which was just a big fenced in yard that had- they called it a chip yard cause it had um, bark chips on the ground and um, I used to go and take this dog out and throw the ball for him and let him run and play and I'd do some TTouch on him and um, it was just- I really enjoyed spending time with him and it was um I had kind of mixed feeling when he got adopted. It was like yeah, I'm glad he got a home but I'll never see him again. (Volunteer A)

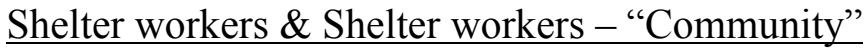

Findings revealed that there was also relational bonding among shelter staff and volunteers.

My dog walking group as several others were social as a group you know had dinners together and um, it just became a you know a connection here other than the dogs who come and go you know, I mean like you can connect with a dog but then it's adopted and you have to make a new connection and so, that's been important to me. (Volunteer B)

And I really liked that, liked being such good friends with everybody that we'd go to each other's houses or go out for drinks, or they'd come to my parties and it was fun to be that tight knit with everybody. And um, also when I was there, they 
were like "Oh go ask __ " and that was a good feeling just to be that in the know on everything. (Volunteer $\mathrm{C}$ )

So, I get to meet people just like me, you know I get to meet the tough guys that still have the sensitive side. And so I get to meet men and women that really want to do the same things I do. And I like that, I like that it's very easy to work with these people. (Staff 1)

The volunteer who helped me was taking out a smaller grey dog and said to me "Isn't she great?" and walked up to me smiling with the dog in her arms like an offering. I reached out and touched the dog's head and agreed. In this moment in the shelter, I felt accepted by my fellow volunteer, and the dog appeared to be a mechanism for the interaction. (Field note, 2010)

Research suggests that companion animals can be catalysts for human interaction (Eddy, Hart \& Boltz, 1988; Irving, 2004; McNicholas \& Glyn 2000; Serpell, J., 2000). Although animals appeared a main reason shelter workers were at the shelter, the presence of the animals still appeared to encourage human interaction. This appeared to be the case during the initial new volunteer orientation. One of the volunteers taking part the training let her two dogs off leash in the lecture room as volunteers settled before for the presentation:

Two dogs were wandering around, coming up to people, and weaving through the chairs that people sat in as they socialized or eating the doughnuts and coffee provided at the entrance. Some people ignored the dogs; others called them over to interact with them. People talked about the dogs as they pet them. (Field note, June 2010)

On the other hand, some volunteers spoke of shelter volunteers not developing relationships or "connections" with other staff or volunteers in the shelter.

Um, I think the people who have difficulty are people who don't have a place to start and who don't have a connection here. Because, it's really hard to walk through the door and look to see what needs to be done. You're not really confident at first maybe to say "Wow there's a ton of laundry I should probably help with that, or somebody needs to make some Kongs, maybe I can do that, or this dog looks really...like he needs some help, I should sit with that dog." I-I 
think that's a gap, I think that there could be umm, I don't know if it be a class or something that would teach people to be a volunteer here. (Volunteer A)

And I think a lot of people come through and they're like "It's so big, there's so many rules, there's so many processes, there's so many volunteers, there's tons of stuff. I'm just this person, I'm just one person" versus "I'm just going to walk around here and make my mark." (Volunteer $\mathrm{C}$ )

There's one volunteer in particular uh, who, I would say has helped the atmosphere a lot. He introduces himself, and talks to other volunteers and you know, talks about his cat and really involves them. Before that, the atmosphere was less friendly among the volunteers, we didn't really socialize that much, so it's better. And maybe part of that is just that I have been here longer now. But when I started, I-I felt all alone (laughter). (Volunteer E)

The foregoing data excerpts illustrate the perceived importance of shelter workers developing a connection between each other, and the disadvantages of being less connected and involved within the shelter community.

\section{Community}

Both volunteers and staff spoke of finding a "common purpose" or goal in their work at the shelter in the repeated phrase:

Um, the staff all seem to be really dedicated you know? It is for the animals you know? It's all about the animals. (Volunteer E)

She states that "the bottom line is, we are here for the animals." (Field note, 2010, Dog Safety and Handling class)

And um, when you get there you are all there for the dogs and I think that's really something that is kind of a uniform saying amongst the people I know is that it's about the dogs. You know, it's not about us, it's not about the rules and all of that. It really is ultimately about the dogs. (Volunteer B)

Thus, the opportunity to meet and connect with other shelter volunteers and staff

who shared similar goals appeared to be an important aspect of shelter work for some.

And I also like that I've been meeting a lot of like minded people who wanna do the same thing and have the same goals as me and I feel like sometimes that's not, sometimes that seems like a harder thing to do than one would think. (Staff 1) 
I was so impressed, I really wanted to be a part of everything and because I started walking with a dog walking team I was brought into that group thing where we all had a common purpose and I got to know people right away... Well I think when you join a dog walking team you're making a commitment, not just to the dogs but to the team of people, that you're going to be there. (Volunteer B)

One volunteer shared her experience of the humane society as open to ideas from staff or volunteer members:

They um, do a really good job of um allowing volunteers to have ideas about programs or things that would improve the live of the animals and letting them run with those ideas. They do a good job of developing community... It's fabulous that they support that kind of um communities, many communities within the bigger community if you will. (Volunteer A)

Acknowledgement of shelter volunteers and staff who played a part in the adoption of animals was provided in volunteer updates via email:

We just HAD to share these wonderful pictures of Miles (formerly known as Roman at OHS) in his new home. Their other cat is also an OHS alumni and has already taken to his new kitten friend. We wanted to thank everyone who had a part in loving him - socializing, cuddling, multiple medical procedures! He is so lucky to have found a perfect new home! (OHS Volunteer email, February 2011)

Community building is stated within the formal organizational mission statement of OHS:

Building a community of compassion is our goal - through adoption of homeless pets, animal welfare legislation, humane education, abuse investigation, and community outreach and leadership. (Oregon Humane Society, para. 11, 2008)

An extension of the concept of community is reflected in OHS's connection with the public. The Oregon Humane Society holds classes that are open to the public that include animal behavior and training as well as a help-line for behavior problems. Youth day camps and activities for children are held in the summer months, and their artwork was visible within the kennel areas itself. 
Over some of the kennels, near the top are fastened brightly colored bandannas with the dogs name on them in paint. I had noticed that a dog earlier that had a red, raw looking spot on his nose with a large white card on his kennel with large font stating that the red spot was present when he arrived, and had been looked at and is being monitored by OHS vets. The kennel contains photos of children posing with him, stating that he was "a hit at camp." (Field note, September 2010)

$\underline{\text { Shelter workers \& the public }}$

The relationship between shelter workers and the public appeared an important aspect of communication within the shelter. Through careful "matching" of adopters and animals, providing an opportunity to "meet" and "get to know" animals as well as providing information about the animals for the public, shelter workers appeared to promote positive connections between the public and shelter animals.

Um, there's a cat we have available right now named Pumpkin. She's a really independent cat, doesn't like to be touched all that much and we have somebody, not a volunteer but somebody from the public actually who comes in every week or every other week and he will put her in a visiting room and he will read to her. (Staff 3)

Um, I'm thinking just recently we had a dog named __ who this choc- this beautiful chocolate Lab came in with almost zero hair because the owners at the time weren't using the right food so the dog just became hairless and sickly and so over the course of a few weeks we had __ on a good diet, hair started growing back. One of the sweetest dogs you'll ever meet too. Very calm, able to get along with kids and other dogs and maybe even cats. And then just seeing that dog get adopted by a very wonderful, wonderful adopter was just great. (Staff 1)

But, there's also been other times when you know, I can't remember specific animals names, but where just you see them interact with them in a visiting room and you see how happy they are about the animal and you can't wait for them to take them home. (Staff, 2)

\section{Dark side of relationships}

The majority of the relationship language observed in the shelter tended to be positive. However, some shelter staff described the "darker" side of human-animal 
relationships, or termination of relationships, which clustered into 3 types. The first type

is "owner released" situations where animals are "surrendered" to the shelter, often

perceived by shelter workers as "broken bonds." Second are animals that have been

adopted out and then returned, and third, violence and neglect and finally, euthanasia.

I feel like a lot of the animals that are there are because somebody wasn't bonded with them you know? (Volunteer C)

You know we've got so many feral colonies and we have so many people who are moving and just leaving cats. (Volunteer B)

Unfortunately a lot of times at the shelter we'll see the surrender reason being a new baby. I've seen that a lot. (Staff 2)

One volunteer perceived some animals that had been released to the shelter by

their previous owners to be "resilient:"

Well you-k it just amazes me that a lot of these animals have- I mean they're allmost of them are owner released, so people have given them up for a variety of different reasons and um, it just amazes me how resilient they can be you know? They're in a strange place and they're scared and nervous and yet they still make connections with the volunteers and the staff that work with them and seem to trust those people even though the people that they loved and lived with and were um, connected to... abandoned them you know? (Volunteer A)

Well, interestingly, cat number one was where I first learned about separation anxiety in animals. And you know, when you think separation anxiety, sometimes you just think of a child who's at school for the first time. They don't like leaving their siblings, they don't like leaving their mother, their father, you name it. They get really tense, they get really scared and they're crying. (Staff 1)

"Returns," or animals that were brought back to the shelter after being adopted

from OHS were also cited as difficult scenarios for shelter workers.

Um, we get a lot of cats that get adopted and are shy and then they get returned because they are shy. That's hard. (Staff 4)

So __ came in and I started working with her again, she got adopted within like three weeks to a couple... long term shelter dogs who you know are here a long time-they're in a home, they come in here, they're here, which she was here three 
months which is significant, go home, they establish a relationship there, then they come back, then their adopted. It's-it's a lot. (Volunteer B)

A project dog might be again, one of the dogs that's been from place to place to place. Okay I've been in the shelter, I've been in this shelter, now I'm- you know, been home and brought back to this shelter, been home again and then brought back to the shelter. Well, obviously something's going on. (Volunteer D)

A "project dog" was described by one shelter worker as a dog that may come to

the shelter scared, need training or arrive to the shelter from a rescue situation that may

need to be worked with to become adoptable.

Um, an easier project dog, puppy mill. Dogs' never been around people. And you tell people, you know what? Dogs' never been around people. This dog may hide under your couch 24 hours a day, seven days a week. Come out to drink water and poop on the floor, and that's it. And if you're okay with that, than take the dog. But you can't think in your mind that two weeks of being in my home's going to be great. It's not gonna work. This is a project dog. This dog may never be normal, is not normal now. You may never have a normal dog.

In the third type of situation, some shelter workers were confronted the "dark side" of human animal relationships often perpetrated by the public. Shelter workers described the emotional toll of confronting the negative aspects of animal-human interactions. These are identified by neglect and violence in the following narratives.

We have to see a very dark side, and because we also know that there's a connection between animal violence leading to eventually domestic violence, we know that there's some very scary people out there because of where we see them start this process that may lead them to violence to fellow human beings. (Staff 1)

Um, overall, I hate to what I feel to be the dark side of humanity sometimes. We get the craziest things happening. And when you're a person who's empathetic toward animals, you really feel some sympathy when you get these cases of such awful abuse and neglect. And we have to see that. We have to see that somebody decided you know, to throw a cat into a storage bin for weeks at a time. We have to see that someone decided to light an animal on fire. We have to see all of these awful things that somebody just decided to randomly do for fun, or just don't have a part of their brain that tells them to stop, or whatever reason. (Staff 1) 
There can be sometimes when it's stressful and straining on a person. There's um, what we call compassion fatigue where your dealing with animals and the life and health, and/or possible death of an animal and it can be really stressful. (Staff 2)

But, I don't know, I think in general knowing how many hardships animals face and how many horrible things happen to them in a lot of ways, from a protective custody situation where someone's being starved to death, to your parents gave you up cause they had a baby, or their moving. I-I would say that I'm like even more on the animal side and even less into people (laughter). (Volunteer C)

The third type of "dark side" contexts includes euthanasia. The Oregon Humane Society does not euthanize animals for space reasons, only for ill health or significant behavior issues. As such, this issue is cited as a reason that shelter workers chose to work at the shelter.

I feel really lucky to work at OHS just because our euthanasia rate is extremely low. I know a lot of county shelters have to euthanize due to um, space constraints whereas we are not under any space constraints or time constraints...So our save rate and adoption rate are great. And for that, it makes me happy and it makes me be able to continue working there. I don't think I'd be able to work in a place where they were euthanizing healthy and adoptable animals. (Staff 2)

One of the-at least the good thing about me coming to OHS is that euthanasia is not taken lightly by this organization. I've never seen a place with such low numbers. So I like that. (Staff 1)

However, euthanasia does still occur, and appeared to be a subject that affected these shelter workers:

And then just my time in 6 years still trying to come to grips with seeing euthanasia, you go through a lot of uh, different emotions over euthanasia. So, that's-that's been pretty tough too. (Staff 2)

And I was waiting for someone to assist me, and I was telling him to sit and lay down he was doing all of this, and so I got a little bit attached. You know? Just in that brief moment and so afterwards I got really emotional because you know, I didn't know this dog. But I just had that brief attachment to him and it made it you know, it made it really difficult. So sometimes that can be really difficult, really painful for, for me. (Staff 3) 
The "darker" relationship language observed within the shelter spoke to the termination of humans-animal relationships ranging from surrenders and returns of animals to the shelter to violence, neglect and death. Some shelter workers described an emotional toll of confronting the negative aspects of animal-human interactions as well as perceiving some of them as "broken bonds" between humans and animals.

\section{CONCLUSION}

This study used the Ethnography of Communication as a descriptive theoretical framework to understand the speech community at the Oregon Humane Society. Through thematic analysis of individual interviews, field-notes and documents, findings revealed an animal-centric culture that included an overarching theme of relational bonding.

There also appeared to be a network of relationships that appeared across the data between: A. Shelter animals and shelter animals B. Shelter workers and shelter animals C. Shelter workers and shelter workers and D. Shelter workers and the public. It appeared that efforts of shelter workers flowed upwards to support an animal-centric culture. Shelter workers appeared to make efforts to bond with shelter animals, and shelter workers formed relationships with each other in part due to these efforts. Based on content discussed in this chapter, Chapter V includes additional interpretations of the finding and links with the literature. This is followed by limitations, contributions and implications for future research. 


\section{CHAPTER V \\ DISCUSSION AND CONCLUSION}

\section{INTRODUCTION}

As described in Chapter I, as the conception of animal shelters have grown in complexity and scope, shelters across the United States are moving toward a model of public education, behavioral training, and community outreach.

The purpose of this study was to understand the culture-sharing group of people working within a Pacific Northwest animal shelter that exemplified the recent model though the Ethnography of Communication. The guiding questions of this study were: RQ1: What are the cultural communication forms performed in the context of the humane society? RQ2: How do shelter workers communicate about companion animals? RQ3: What cultural meanings are instantiated through communication in this context? Each research question addressed three main lines of inquiry for this study. The first, (RQ1) addressed the communicative forms that comprise speech events, acts and situations (Hymes, 1962). This question addressed the structure of communication within the shelter. The second (RQ2) addressed the process of how shelter workers communicate, while the third (RQ3) research question addressed the context or emergent meanings of shelter workers communication.

Research design included eight individual interviews with Oregon Humane Society staff and volunteers, 40 participant observation hours within the shelter and analysis of OHS documents and artifacts. 
Based on the findings discussed in Chapter IV, this chapter discusses the interpretations of the findings and relevant links with the literature. This discussion is followed by the limitations and contributions of the study including implications for future research. In examining the community of speakers at the Oregon Humane Society through the communication forms of verbal, nonverbal and written, it appeared that they held shared knowledge and beliefs regarding the shelter animals they interacted with.

One of the examples of a common communication form enacted within the shelter is the personification of an animal by using "I." Shelter workers speaking for the animals they interacted with appeared a feature within previous studies of animal shelter culture. Harbolt (2003) argues that shelter animals are "stripped" of their previous definitions and meanings that their owners had created for them, and their biography is reconstructed by shelter workers.

Phillipsen (1992) discusses the "sacred object" of a community, or what the community values. The sacred object of the community of speakers appeared to be bonding, particularly with companion animals. The next section will discuss and expand upon relational bonding using an extant theoretical model from interpersonal communication as a way to further elucidate the concepts of the bonding process. 


\section{INTERACTION STAGES IN RELATIONSHIPS}

The relationship network among shelter workers, animals and the public appears to follow Mark Knapp's (1992) model of interaction stages in relationships characterized by different patterns of communication. This model was initially developed to explain human interpersonal relationships, their development and decline as well as relational maintenance. Knapp's model includes ten stages of interaction. The relationship development or "coming together" stage includes initiating, experimenting, intensifying, integrating and bonding. The de-escalation or "coming apart" stage of the model includes differentiating, circumscribing, stagnating, avoiding and terminating.

Echoing Knapp, Duck (1985) describes relationship development as a process and states that those who are getting to know one another seek to "match" themselves up satisfactorily with others on the basis of a range of factors that include physical appearance, attitude and nonverbal information. Often, this information is based on inferred characteristics, and judgments are refined as more information is available.

The majority of the interaction stages identified within shelter communication fell into relationship development, or the "coming together” stages of Knapp's model. Except for the "terminating" stage, I could not speak to the other relational decline stages due to the fact that shelter workers rarely viewed the "coming apart" stage of Knapp's model between animals and the public. For example, shelter workers were witness to animal "returns," "relinquishment" as well as occasionally the darker side of relationship deescalation such as violence or abuse. In this analysis, stages of coming together and coming apart are detailed and links made where appropriate. 
In the initiating stage of relationship development, communicators are attempting to show that they are pleasant and likable. One volunteer used the metaphor of a dance to describe this stage of relationship development with a shelter animal:

But I love that little dance that you do at first with a dog, to let them read you and you read them (Volunteer B)

At the same time during this stage, individuals are attempting to reduce uncertainty to gain information of mood or interest toward themselves. This stage includes careful observation of the other including exchanging greetings. In shelter literature it has been noted that there is some difficulty of "reading" the personality or behavior of an animal within the shelter setting, as both animals and people may not act exactly as they would at home (Irving, 2004; Alger \& Alger 2003). Shelter workers first attempted to reduce uncertainty about the animals they worked with in various ways which included trainings, mentoring of new volunteers, classes and visual aids of dog non-verbal behavior (Appendix F).

Shelter workers then appeared to engage in reducing this uncertainty for members of the public in several ways. For example, "kennel cards" were displayed on the kennel of each shelter animal. These often included perceived likes or dislikes or behavior traits of the animal:

He is a little worried about all the sights and sounds of city life, so it will take him a while to adjust to his surroundings, as he had a rough start to his life. __ will need a home with someone who is willing to exercise and play with him, as running around on his own in a yard isn't for him (he's proven himself to be an excellent fence hurdler!). (OHS kennel card, June 2011)

Shelter workers are also encouraged to engage with animals in view of the visiting public. For example, interacting with dogs in their kennels created a scene of 
interaction and engagement for the public walking by. Volunteers working in the cattery were encouraged to "sit" with cats in the clear glass colony rooms, which were visible to the public, either grooming them or "socializing" with them. These behaviors perhaps modeled the way the shelter animal might act with a potential adopter:

This creates a favorable impression for the public, as they may walk by and see a dog interacting with someone they then can see that scene in their home, thus potentially making that dog adoptable. This also may approximate an environment that the dog may have experienced in their former life, of hanging out with a person in a home-like environment. (Field note, September 2010)

This behavior may also reduce uncertainty for a member of the public considering adoption of a shelter animal. Potential adopters may also reduce uncertainty about an animal by asking questions or petting/holding an animal within the process of interacting with it in either the "Meet and Greet Room" or "Get Acquainted Room" (see Speech Event II).

Finally, this stage may also include scanning our own stereotypes of the other. For example, one volunteer described this stage as she "introduced" a member of the public to a shelter dog. The shelter worker, who had already "gotten to know" the dog became frustrated when members of the public appeared to display preconceived notions about the type of dog they were going to be "meeting."

So, I'd always walk them over to meet __. I mean, nine times out of ten I wouldn't tell them what they what we were going to see. I would just say "I know ever dog in the shelter, hands down, this is the best, best dog in the shelter. And has been the best dog for months of any dog that has come into the shelter." And they'd be like Oh, and then I'd be like asking them their situation and they'd tell me and I'd thinking like this is perfect. Walk around the corner, "This is "Oh she's a Pit Bull, we would never adopt a Pit Bull." ...was hands down the best dog in the shelter, and she would not get-no one would look at her cause she was a Pit Bull. (Volunteer C) 
The initiating stage of relationship development was also observed among shelter workers. As a new volunteer, I observed the initiating stage of relationship development first hand:

The volunteer who helped me was taking out a smaller grey dog and said to me "isn't she great?" and walked up to me smiling with the dog in her arms like an offering. I reached out and touched the dog's head and agreed. (Field note, September 2010)

Another volunteer noted the positive difference in the shelter environment when shelter volunteers initiated contact with others:

There's one volunteer in particular uh, who I would say has helped the atmosphere a lot. He introduces himself, and talks to other volunteers and you know, talks about his cat and really involves them. Before that, the atmosphere was less friendly among the volunteers, we didn't really socialize that much, so it's better. (Volunteer C)

In the experimenting stage, communicators begin the process of discovering new things and gaining further information about the other individual. The shelter appeared to facilitate both stages of initiating and experimenting between potential adopters and shelter animals by encouraging them to visit the "Get Acquainted" and "Meet and Greet" rooms in the shelter. This appeared to allow potential adopters space to engage in "sizing up" or the attempt to obtain information about others while reducing uncertainties (Knapp, 1992).

In addition, within the experimenting stage, adopters may be searching for potential similarities (Knapp, 1992). Within shelter literature, this stage appears to take place as potential adopters are spending time with an animal before adoption. Animals may communicate liking within the shelter through their bodies "Dogs stay close, wag 
their tails, and relax their faces and ears. Animals can also show interest, if not affection through attentiveness" (Irving, 2004).

During the third Intensifying stage, there is active participation within the relationship, such as creating terms of endearment or direct expressions of commitment. In relation to the public, a volunteer who assisted in an adoption of a shelter dog demonstrated the intensifying stage:

This guy came in and met two or three times, fell in love with fell in love with him and on Christmas Eve last year... got adopted, snow was up to here, we were closing early, he comes in and adopts this dog on Christmas Eve. (Volunteer D)

Among shelter workers, active participation in relationships between some shelter workers that originally developed within the shelter, appeared to continue outside the shelter, exemplifying the intensifying stage:

And I really liked that, liked being such good friends with everybody that we'd go to each other's houses or go out for drinks, or they'd come to my parties and it was fun to be that tight knit with everybody. (Volunteer C)

My dog walking group as several others were social as a group you know had dinners together and um, it just became a you know a connection here other than the dogs who come and go you know, I mean like you can connect with a dog but then it's adopted and you have to make a new connection and so, that's been important to me. (Volunteer B)

Because the shelter appeared to be a transitory environment for shelter animals, the ability of a shelter worker to intensify an existing relationship with a shelter animal did not always take place:

I think the ones that stay around longer, you know, obviously are the ones that make more of an impression. Cause we spend more time with them and they're not out the door right away. (Staff 3) 
In the stages of Integrating and Bonding, the relationship has progressed to a stabilization of the relationship. The word bond was a term discovered across all data types:

And, you know there have been moments where I have felt a bond between this creature, this animal and myself a human being. (Volunteer E)

This lady would appreciate a quiet home where she will have time to adjust and bond with her new family. (Volunteer email, February 2011)

And I consider myself very fortunate because I have friends who have cats and they're just cats. They happen to be pets that are in the household but there doesn't seem to be that bond. And I think if you take-if your lucky and you take the time to try and establish something with the animal, that's really special. (Volunteer E)

Bonding is described as a relationship stage in terms of an extension of

integrating, or a commitment to the relationship (Knapp, 1984), or intimacy based on “complex behavioral interdependence" (Trenholm \& Jensen, 1996, p. 334). An example

of the bonding process between a shelter volunteer and an animal is described in detail:

There's a certain look that a dog has when they look at you from the perspective of "what are you going to do to me, I don't like you, I don't trust you." And then after a while you go through a phase where they look at you all the time cause they don't want-you're going to be staring at them, so you don't look at them, but they're staring at you cause their like whatta you doing. Then there's a time when they're not looking at you, and they're getting to know you and then there's a final time like maybe they're in your lap or their jumping up, or their doin' things where they have a totally different look and you go there's the bond. And you can see it in their eyes, I trust you. Um, For example a dog'll lay down next to you, put his back up against you. Well, he's no longer looking at you, it's a very vulnerable position, but he knows that you got his back. Literally, you got his back covered, if something' comes this way your going to take care of it, or alert him. So when-when I'm showin' dogs to clients and their sitting in there and finally the dog just, overwhelmed, or whatever the case is comes down and just bonk, back up against them that's a good sign. The dog trusts you. And that's part of the bond. (Volunteer D) 
The latter stages of bonding and integrating appear to be similar to the adoption of an animal and the integration of the animal into the individual's home. Shelter workers who had adopted shelter animals described this stage in their own experiences:

And _ was the one, he came in um, and then he got adopted by a family with children and then he was returned because he wasn't good with children. So I adopted him...I really liked him. He's a hugger, so when he gets nervous he'll come up to a person and climb up and snuggle. And that doesn't happen very often with cats so it's hard not to fall in love. And he got adopted, that was great, but when he got returned he was mine. My cat. (Staff 3 )

During my nine months at OHS, I was unable to address the de-escalation, or coming apart stages of Knapp's model, as this study did not include interviews with the public, and de-escalation stages were not mentioned by shelter workers within interviews. However, shelter workers did touch upon the final "termination" stage of Knapp's model.

\section{DARK SIDE OF RELATIONSHIPS}

Relational dissatisfaction, dissolution, violence and conflict can also be features of relationships. The darker side of relationships, particularly relationships between human and animals was discussed by shelter workers, and appeared as a topic within shelter literature. Duck (1994) argued that the some of the darker sides of relationships may be a feature of relational stages, and that conflict within relationships is not necessarily an inherently negative aspect, but can be a challenge within relationship building (Duck, 1994). For shelter workers however, the dark side of relating appeared to present itself as a primarily negative aspect of human-animal relationships. It is thought that "harming those who have little power to protect themselves from harm is another source of darkness" (Spitzberg \& Cupach, 1998, p. xiv). 
From the shelter worker's perspective, the "termination" stage of Knapp's model which can include aspects of the dark side of relating, appeared to be viewed by shelter workers as generally negative, as this stage resulted in animals being returned to the shelter, death of an animal or neglect.

For example, not giving the relationship between new animal and adopter time to form a bond at all appeared to be a factor of relinquishment. This is reflected in recent shelter literature. In one study of animal's shelter relinquishment, forty percent of adopters in the study returned an animal within a week (Mondelli et al., 2004). The issue of "returns" was also mentioned by shelter workers:

I feel like a lot of the animals that are there are because somebody wasn't bonded with them you know? (Volunteer C)

So __ came in and I started working with her again, she got adopted within like three weeks to a couple... long term shelter dogs who you know are here a long time-they're in a home, they come in here, they're here, which she was here three months which is significant, go home, they establish a relationship there, then they come back, then their adopted. It's-it's a lot. (Volunteer B)

Violence was touched upon explicitly as a "dark side" of some human-animal relationship by one shelter staff member:

Um, overall, I hate to what I feel to be the dark side of humanity sometimes. We get the craziest things happening. And when you're a person who's empathetic toward animals, you really feel some sympathy when you get these cases of such awful abuse and neglect. And we have to see that. We have to see that somebody decided you know, to throw a cat into a storage bin for weeks at a time. We have to see that someone decided to light an animal on fire. We have to see all of these awful things that somebody just decided to randomly do for fun, or just don't have a part of their brain that tells them to stop, or whatever reason. (Staff 1)

According to the literature, the decision to euthanize or surrender an animal can be a difficult one for the adopter (Harbolt, 2003). Recent studies have examined quantitatively the reasons why adopters relinquish animals (Mondelli et al., 2004; Salman 
et al. 2000; Salman et al. 1998). Adopters cite moving and behavior of the animal as

common reasons for relinquishment. Recent literature also suggests that shelter workers tend to be critical of the public's reasons for relinquishment of animals (Taylor, 2007).

I can't think of a situation unless a-I was a hermit, had no friends, had no family, um and had to go into the hospital for a terminal illness. Okay, there would be a reason to give up your animal. (Volunteer C)

\section{Euthanasia}

Euthanasia was a aspect of the relationship termination stage of that informants could speak directly to as it was an experience internal to the shelter. Previous shelter studies weighed heavily on the topic of euthanasia and the emotional impact it can have on shelter workers in mostly in high-kill shelters due to space or simply lack of suitable adopters as well as the decision-making on the part of shelter workers involved with euthanasia (Arluke, 1991; Harbolt, 2003).

Due to this, I was interested in whether this would be a topic that workers would broach working in a shelter where animals were not euthanized for space reasons. My interview questions did not include euthanasia, and I did not bring it up unless the interview participants mentioned it first. Even in a shelter such as OHS where animals were not euthanized due to space limitations, euthanasia did occur and still appeared to be a traumatic event for shelter workers.

And then just my time in 6 years still trying to come to grips with seeing euthanasia, you go through a lot of uh, different emotions over euthanasia. So, that's-that's been pretty tough too. (Staff 2)

But a couple of weeks ago I was scheduled back there on a Friday and Saturday which are really busy days for owners to bring in their animals for euthanasia cause it's the weekend, it's when they have time off. And um, I remember, it was like, a little Rat Terrier or something. He was just old but he was still coherent. And I was waiting for someone to assist me, and I was telling him to sit and lay 
down he was doing all of this, and so I got a little bit attached. You know? Just in that brief moment and so afterwards I got really emotional because you know, I didn't know this dog. But I just had that brief attachment to him and it made it you know, it made it really difficult. So sometimes that can be really difficult, really painful for, for me. (Staff 3)

Other volunteers expressed some ambivalence about euthanasia:

I have done it, and um, sometimes, sometimes you think this is definitely a good thing that I'm doing because this animal is really in pain and it needs to be done, and you start to believe that their fate is worse than death. And then there's sometimes where you're just not sure that this is the right choice. And you're not quite sure especially when your in a lower position how to make this process not happen, or how to explain why you think that this doesn't need to happen. (Staff 1)

One shelter worker expressed responsibility and guilt for the death of an animal, attributing the euthanasia to the fact that he had not "worked" with the animal as he thought he should:

And while I was sitting in the kennel they went to get her for euthanasia. And I said right then I will never put anything ahead of working with a dog. Because I felt guilty you know? If I had put more hours and more time, you know more focus on her rather than you know, show a dog, show a dog, show a dog, 50 different things, scoop a kennel, move some bags of dog food around. She wasn't the priority. And she got euthanized. And I felt bad and was like whenever anyone says "would you work with" that's all I'm gonna do. If I don't get anything else done, I'm gonna work with that dog and I'm gonna make sure that anytime I come in I work with that dog cause I felt the dog had-had a chance. Had she been worked with enough. Uh, and I really felt like you know, I dropped the ball. (Volunteer D)

Euphemisms are often used within cultural communities in order to cope with issues or topics that are taboo or difficult to deal with, and are not uncommon in American society (Pound, 1936). Euphemisms for death are often used to "soften the verbal impact" of the meaning (Saville-Troike, 2003). Within individual interviews the word death was not used in reference to euthanasia, rather it was found that shelter workers often used euphemisms to describe euthanasia. 
Some words were formal language of the shelter such as notes indicating that animals were not to be euthanized represented by the acronym "No PTS" or "No Put to Sleep." The room where euthanasia was carried out is named the "ER" room. Informal speech was also noted, primarily during individual interviews.

Signed off is the code word for euthanasia. Dog's been signed off. (Volunteer 2)

Knowing that they're in pain, they are suffering in some way and solving that pain. But being the one that has to um, do that can just really wear you down. But being the one that has to um, do that can just really wear you down. (Staff 3 )

You'll hear that on the radio, I need help back in ER. That's not a good thing. That means y'know, they've got something to do back there. (Staff 2)

I think we do much less of that than when I first started here, and we didn't do very much then. But you know, occasionally you would have a dog put down. (Volunteer D)

She's goin'. And you know what that means, and I'm like oh man. (Volunteer 2) OHS is considered a "no kill" organization and as noted earlier, this was perceived as a positive by shelter workers. It appeared that although the justifications for humanly euthanizing an animal were carefully considered and weighed by shelter workers, the opportunities for relationships with the animals that the workers were able to build due to some animals' long term stays appeared to make euthanasia possibly as equally difficult as in higher-kill shelters.

It appeared that the shelter workers promotion of bonding between an adopter and shelter animal was crucial in possibly preventing the return of an animal to the shelter 


\section{DISCUSSION}

The findings of this study revealed an animal-centric culture that included an overarching theme of relational bonding. The relationship communication present within the shelter setting appeared to support the overarching promotion of bonding. Shelter workers appeared to make efforts to bond with shelter animals, and shelter workers formed relationships with each other in part due to these efforts. Further, shelter workers acknowledged the significance of animal-animal bonding, and worked to ensure as much as possible, bonding between the public (adopters) and animals.

\section{Promotion of bonding}

It appeared that the relationships between shelter workers and shelter animals occurred in the service of bonding, often with the shelter animals they cared for. Shelter workers first attempted to reduce uncertainty about the animals they worked with in various ways which included trainings, mentoring of new volunteers, classes and visual aids of non-verbal behavior (Appendix F). Further, "getting to know" and building relationships with shelter animals encouraged trust and relational bonding between shelter workers and shelter animals.

Shelter workers also attempted to promote a similar kind of relationship with a potential adopter. In order to reduce uncertainty about shelter animals, the shelter informed the public about shelter animals through the use of kennel cards, provided a place for animals and people to get to know one another in the "Meet and Greet" and "Get Acquainted" rooms, screened potential adopters, provided classes for the public, as well as prepared and presented shelter animals in the most favorable light including "modeling" what an animal might be like with an adopter within public view. 
The importance of making a correct "match" with an adopter appeared to be carefully considered and prepared for. For example, shelter animals were not placed "on the floor" for adoption until they were considered adoptable by the public. When shelter animals appeared to "shy" or "scared" or "aggressive" they were often worked with by a shelter volunteer or staff member to make them more sociable and therefore, more adoptable.

So then you try to socialize them, and work with them and earn their trust. (Volunteer 3)

This included working with animals may come to the shelter scared, need training or arrive to the shelter from a rescue situation that may need to be worked with to become adoptable. One volunteer described some of the dogs he worked with as "project dogs" that fell into these categories. This often happened "in the back" or away from the public.

Sociologist Erving Goffman (1959) uses the stage as a metaphor for social interactions between individuals within communities. Goffman posits there is a "structure to social encounters" (Goffman, 1959, p. 128) and that social actors project themselves before a particular set of observers. Front stage is used to describe the way an individual may act when they know they are being watched. Back stage describes how social actors may behave in private or with a community of peers in a more unrestricted way. Within organizations, these terms can describe the public and private areas of spaces. The spatial elements of the shelter "back there" and "up front" were terms used by shelter workers and identified in my field notes to denote the public and private or shelter worker portion of the shelter. 
Every business has it's own buzz words and things you know. "In the back" you know? This is in the back, like we're in the back now. "On the floor" is out there (points toward the kennels), uh "up front," front desk. (Volunteer D)

Well how the process works on the cats side is we'll get in a cat, it'll get vac'sed and put away in a kennel in the back and then probably depending on the amount of animals we have at that time, they'll maybe sit for a day or two in the back and you sort of get to know them. (Staff 3)

She tells a story stating the importance of reading the kennel cards, that in the old building there was only a front/public section and that volunteers and staff had to write "cryptic notes" attempting to communicate the temperament and behavior of the dogs on the front cards. (Dog Safety and Handling Class, 2010)

It's a really good feeling when you know that there's a dog that you know, been in the back. And the back is hidden away from the public. And it's like this dog is in a situation where he's being naughty as they say and would bite people and is a risk, and to work with a dog enough to where the dog goes home and you know, you come in and the dog is either going home or the dogs already gone home. (Volunteer D)

Animals that had been surrendered had to be screened for health and behavior before they could be placed "on the floor" for adoption and viewing by the public. This activity happened "in the back" or within the inner area of the shelter. Euthanasia also was an activity that happened "in the back" and as an issue that was often difficult for shelter workers, "the back" was a phrase that in the words of shelter volunteer, substituted for the act itself:

You'll hear that on the radio, I need help back in ER. That's not a good thing. That means y'know; they've got something to do back there. (Volunteer D)

The relationship network between shelter workers, animals and the public also appeared to create a sense of shared community. Within previous animal shelter literature, it appears that shelter workers are spinning their wheels in terms of keeping up with the sheer volume of animals (Harbolt, 2003). Within the cultural community at OHS, shelter workers appeared to have the time and resources to develop relationships 
with animals. The following narratives describe the relationship between an animals'

length of stay at the shelter and shelter workers attachment to the animal:

I think it would be very hard to become as attached as we do to our longer residents knowing that they have such-and-such amount of time before they get euthanized. Yeah, I would have to say that you would probably have to feel a little bit more detached from the animals you're working with if you were in that kind of environment. Otherwise how would you, how would you work there for a long time? (Staff 3)

You know, not everybody gets really attached to certain dogs because they didn't have the opportunity to really spend time with them. (Volunteer C)

Um, yeah, we had a little cat uh, __ like a petite, she was a full-grown cat but she was a very petite cat. Like maybe only 5 pounds, um, and she was in our care for several months. Because while in our care she contracted ringworm. It's pretty easily remedied but it takes a long time. It sticks around for several weeks. The treatment period lasts a long time so myself, and many of the staff grew really attached to her cause we were taking care of and treating this cat fer months and months and months. Um, waiting her for her to be ready for adoption. And it turns out the medication she was taking to uh, get rid of the ringworm, uh, caused her to go into early onset renal failure, or kidney failure... So, she just, she sat there for a while and um, unfortunately her kidney values just kept deteriorating, it was-we weren't able to regulate um, the disease with food like we can with some cats, so unfortunately she has to be euthanized also. So, that made a big impression on me cause that was the cat that um, as a team we all grew really attached to. And it was hard to see her go, you know? (Staff 2)

Um, I think maybe the length of stay is something that has to do with it. Um, the longer they're there the more time you have to grow attached to them. If you're cleaning their kennels and medicating them every day then you get an attachment to them. Otherwise like kittens, I don't grow an attachment to them cause they come in, they get screened, spayed or neutered before even, there's like a two or three day turn around period on kittens. You know, they justthey come and go. But any cats with special needs or um, usually it's just special needs. They are on special food, require medication or are old. Then they tend to stick around a little bit longer. Um, and so we grow attached to them. (Staff 3)

The animals that stayed in the shelter for a length of time appeared to bind the staff together, creating a sense of community. Whereas in previous animal shelter literature, euthanasia brought shelter workers together into an "inner community" 
(Arluke, 1991) at the Oregon Humane Society the "common purpose" of shelter workers, of placing animals in suitable homes through a successful match appeared to bind workers to each other.

\section{Shelter cultural changes}

The increased complexity of some animal shelters has allowed humane societies to focus efforts on preventing overpopulation of animals and public awareness (Shultz, 1924). Many shelters have transitioned from places of "last resort" to often complex organizations that contain a variety of public services that include adoption, sterilization and behavioral counseling (1985, Arkow). As reflected in recent literature, this cultural shift was noted by shelter staff:

A lot of it is we're replacing a lot of old negative language used to describe us. We're not an animal jail, we're a humane society, we're a shelt- you know we don't even really say shelter anymore, we just kind of say we are a humane society... But yeah, I guess a lot of it is just getting rid of- that we are not just going out there and grabbing animals off the street. We are not just going out there and grabbing your pets that happen to be walking around. We are really trying to help. And so a lot of the jargon that is used is just to make that- to really depict us properly in what we actually do. (Staff 1)

An initial expectation that I had before the study was to possibly discover themes of rescue, disenfranchisement or a more politicized atmosphere and discourse surrounding the problems of animal welfare or rights. What was overwhelmingly present was the theme of relationships and the overarching key theme of relational bonding.

I did not expect to find this as the overarching dominant theme when I began the study. Observed shelter communication was not devoid of themes surrounding the political aspects of animal welfare. For example, on the OHS website there are links to current advocacy campaigns targeting animal welfare and a story featured in the Oregon 
Humane Society Magazine details the attempted prosecution of a dog owner who had abused his dog later rescued by the shelter (Koppes, 2010). However, these were not themes that were overwhelmingly present in the communication observed for this study.

\section{LIMITATIONS}

One of the limitations of this study is that it was focused on one area of the shelter. I did not conduct interviews, or include observation notes of specific public activities or discourse within the shelter. As the public and community is an integral part of the humane society, this was a limitation as I was not able discover the public's understanding of shelter animals and the shelter itself.

Another limitation was that of total time spent at the shelter and areas observed. This study was limited to under a year and 40 observation hours and interviews with 8 volunteers and staff. Finally, the shelter area where the majority of observations occurred is only one part of the larger network of services that make up the Oregon Humane Society.

\section{CONTRIBUTIONS}

This ethnographic study of the cultural sharing group of the Oregon Humane Society sought to understand shelter workers. Scholarly inquiry into human animal interactions is a currently a field in development (Wolfe, 2009) and animal shelters and humane societies are fairly recent cultural institutions, existing in the United States for less than 150 years (Arkow, 1985). Understanding how and why a culture relates to animals can be a window into an aspect of that distinct culture. 
The study of interpersonal relationships has generally focused on human-human relationships. However, little research has been conducted within the field of interpersonal communication looking at interspecies interactions. As more than half of households in the United States live with a companion animal (Risley-Curtis et al., 2006) there appears to be gap in research and understanding of human-animal communication and interactions. This study may shed light on these interactions, and they ways in which people view, value and relate to companion animals.

\section{FUTURE RESEARCH}

Future research might be completed within the other areas of this shelter in order to obtain a more holistic picture of a complex organization. For example, a study of other departments such as Humane Investigations or the Education department might uncover different themes than those found in the shelter areas. As a theme of relationship development and the darker side of relationships were discovered within this study, a future study might look at the public interaction with animals more closely to understand further the relationship building and potential breakdown that leads to surrender or relinquishment to a shelter.

As a new volunteer within the shelter, I was unable to speak to the de-escalation of Knapp's (1980) relationship model. The specific causes of violence, neglect or the relinquishment of animals to the humane society was not known to most shelter workers, as they were often only witness to the final stage, or the "terminating" stage of the model. As shelter literature suggests that a cause of relinquishment of animals to the shelter may be the lack of a bond forming between the companion animal and adopter (Mondelli et 
al., 2004) conducting a study that looks specifically at this model might be beneficial for both shelters and adopters to understand these causes. 


\section{REFERENCES}

Arkow, P. (1999). The Evolution of Animal Welfare as a Human Welfare Concern in F. R. Ascione \& P. Arkow (Eds.) Child Abuse, Domestic Violence and Animal Abuse: Unlinking the Circles of Compassion for Prevention and Intervention p. 19-35 Indiana: Perdue University Press

Arkow, P. (1985). The Humane Society and the Human-Companion Animal Bond: Reflections on the Broken Bond. Symposium on the Human-Companion Animal Bond. Veterinary Clinics of North America: Small Animal Practice. Vol. 15, No 2

Arluke, A. (1991). Coping with euthanasia: A case study of shelter culture. Journal of the American Veterinary Medical Association. Vol. 198

Arluke, A. (1994). Managing Emotions in an Animal Shelter. In A. Manning and J. Serpell (Eds.), Animals and Human Society: Changing Perspectives. (pp. 145165) London: Routedge.

Blumer, H. (1954). What Is Wrong With Social Theory? American Sociological Review. Vol. 19, No. 1.

Briggs, C. (1986). Learning How to Ask: A sociolinguistic appraisal of the role of the Interview in social science research. Cambridge University Press: Cambridge

Buettinger, C. (1997). Women and Antivivisection in the Late Nineteenth-Century America. Journal of Social History, Vol 30, No4 pp. 857-872

Cresswell, J. (1998). Qualitative Inquiry and Research Design: Choosing Among Five Traditions. Thousand Oaks: Sage

Duck, S. (1994). Stratagems, spoils and a serpent's tooth: On the delights and Dilemmas of Personal Relations in Cupach, W., \& Spitzberg, B., (Eds) The Dark Side of Interpersonal Communication. New Jersey: Lawrence Erlbaum Associates, Inc. 
Duck, S. (1985). Social and Personal Relationships in Knapp, M. \& Miller G. (Eds.) Handbook of Interpersonal Communication. Beverly Hills: Sage

Eddy, J., Hart, L.A., \& Boltz, R.P. (1988). The effects of service dogs on social acknowledgement of people in wheelchairs. Journal of Psychology, 122, 3945.

Ekman, P. \& Friesen, W. (1974). Nonverbal behavior in psychopathology in Friedman, R. \& M. Katz (Eds), The Psychology of Depression: Contemporary Theory and Research. Washington D.C.: Winston \& Sons p. 203-232

Fetterman, D. (1989). Ethnography: Step by Step. Applied Social Research Methods Series Vol 17. Newbury Park: Sage

Furst, G. (2007). Without words to get in the way: Symbolic interaction in prisonbased animal programs. Qualitative Sociology Review, 3(1), 96-109

Geertz, C. (1973). The Interpretation of Cultures. New York: Basic Books, Inc.

Goffman, E. (1959). The Presentation of Self in Everyday Life New York: Doubleday

Gumpertz, J.J. \& Hymes, D. (Eds.). (1972). Directions in Sociolinguistics: the Ethnography of Communication. New York: Holt, Rinehart \& Winston.

Hall, R. (1999). The Link's Direct Connection to Child Protective Services in F. R. Ascione \& P. Arkow (Eds.) Child Abuse, Domestic Violence and Animal Abuse: Unlinking the Circles of Compassion for Prevention and Intervention p. 109-119 Indiana: Perdue University Press

Hammersley, M., and Atkinson, P. (1995). Ethnography: Principles in Practice. $2^{\text {nd }}$ Edition. New York: Routledge

Harbolt, T.L. (2003). Bridging the Bond: The Cultural Construction of the Shelter Pet. Purdue University Press 
Humane Society of the United States (2009). HSUS Pet Overpopulation Estimates Retrieved from http://www.humanesociety.org/issues/pet_overpopulation /facts/overpopulation_estimates.html

Hymes, D. (1974). Foundations in Sociolinguistics: An Ethnographic Approach. F Philadelphia: University of Pennsylvania Press Inc.

Hymes, D. (1964) The Ethnography of Communication. American Anthropologist, pp. 1-34

Hymes, D. (1962). The Ethnography of Speaking in Gladwin, T. \& Sturtevant, W.C. (Eds.), Anthropology and Human Behavior, The Anthropology Society of Washington, pp. 13-53.

Ingold, T. (1994). From Trust to Domination: An Alternative History of Human-Animal Relations. Animals and human society

Irving, L. (2004). If You Tame Me: Understanding Our Connections With Animals. Temple University: Philadelphia

Irving, L. (2002). Animal Problems/People Skills: Emotional and Interactional Strategies in Humane Education. Society \& Animals 10, 63-91

Johnson, J., (1990). Selecting Ethnographic Informants. Qualitative Research Methods Series 22. Sage Pubs, CA

Johnstone, B. (1990). Stories, Community, and Place: Narratives from Middle America. Indiana University Press, Bloomington Indiana

Kirk, J., \& Miller, M., (1986). Reliability and Validity in Qualitative Research. Qualitative Research Methods Series 1. Sage Pubs, CA

Koppes, R. (2010). Midnight's Long Run. Oregon Humane Society Magazine. Vol. 39(3), pp. 15 
LaFrance, C., Garcia, L. J., \& Labreche, J. (2007). The effect of a therapy dog on the communication skills of an adult with aphasia. Journal of Communication Disorders, 40(3), pp. 215-224.

LeCompte, M. and Goetz, J. (1982). Problems of Reliability and Validity in Ethnographic Research, Vol. 52, No. 1 pp. 31-60

Lincoln, Y., Guba, E. (1986). But is it rigorous? Trustworthiness and authenticity in naturalistic evaluation. In D.D. Williams (Ed.), Naturalistic evaluation, pp. 7384. New Directions for Program Evaluation, 30. San Francisco, CA: Jossey-Bass.

Lincoln, Y., Guba, E. (1985). Naturalistic Inquiry. Sage Publications, Newbury Park, CA.

Lofland, J., Snow, D., Anderson, L., Lofland, L. (2006). Analyzing Social Settings: A Guide to Qualitative Observation and Analysis. Fourth Edition. Belmont CA: Wadsworth/Thompson Learning

Luborsky, M. (1994). The Identification and Analysis of Themes andPatterns. In Jaber F. Gubrium \& Andrea Sankar (Eds.) Qualitative Methods in Aging Research. Sage Pubs. Thousand Oaks, CA.

Mangum, T. (2002). Dog Years, Human Fears in Representing Animals. In N. Rothfels (Ed.), Representing Animals (pp. 35-47). Bloomington: Indiana University Press

Mason, J. (2005). Civilized Creatures: Urban Animals, Sentimental Culture, and American Literature, 1850-1900. The John Hopkins University Press. Baltimore, Maryland

Maxwell, J. (2005). Qualitative Research Design: An Interactive Approach. Second Edition. CA: Thousand Oaks Sage Publications

McCracken, G. (1988). The Long Interview: Qualitative Research Methods Series, Vol 13. Newbury Park, CA: Sage 
McKay-Semmler, K. (2007). The No-Kill Shelter: Redefining Dignity and the Humane Society. Conference paper/unpublished manuscript

McNicholas, J., Collis Glyn (2000). Dogs as catalysts for social interactions: Robustness of the effect. British Journal of Psychology. 91, pp. 61-70

Mondelli, F., Prato Previde, E., Verga, M., Levi, D., Magistrelli, S., \& Valsecchi, P. (2004). The bond that never developed: adoption and relinquishment of dogs in a rescue shelter. Journal of applied animal welfare science JAAWS, 7(4), 253-266.

Oregon Humane Society (2010). OHS New Volunteer Handbook 2010

Oregon Humane Society (1915). The Oregon Humane Society; The Oregon Humane Society (member American Humane Association) containing abstract if the state laws, the constitution of the society, what the Oregon Humane Society is doing, information to organize a society, humane work throughout the world, general intelligence.

Oregon Humane Society (2008). Retrieved April 28, 2010 www.oregonhumane.org

Patton, M. (2002). Qualitative Research \& Evaluation Methods $3^{\text {rd }}$ Edition. Thousand Oaks, CA: Sage

Peterson, M., Pike, K. (2002). Emics and Etics for Organizational Studies: A Lesson in Contrast from Linguistics. International Journal of Cross Cultural Management, 2, 5.

Phillips, M. (1994). Proper names and the Social Construction of Biography: The Negative Case of Laboratory Animals. Qualitative Sociology Vol. 17 No. 2

Philipsen, G. (1992) Speaking Culturally: Explorations in Social Communication. Albany, NY: State University of New York

Pound, L. (1936). American euphemisms for dying, death, and burial. American Speech 11(3): 195-202 
Risley-Curtiss, C., Holley, L., Cruickshank, T., Porcelli, J., Rhoads, C., Bacchus, D., Nyakoe., S and Murphy, S. (2006). "She Was Family:” Women of Color and Animal-Human Connections. Affilia, pp 21; 433

Salman, M. D., Hutchinson, J., Ruch-Gallie, R., Kogan, L., New, J. C., Jr., Kass, P. H., et al. (2000) Be- havioral reasons for relinquishment of dogs and cats to 12 shelters. Journal of Applied Animal Welfare Science, 3, pp. 93-106.

Salman, M. D., New, J. C., Jr., Scarlett, J., Kass, P., Ruch-Gallie, R., \& Hetts, S. (1998). Human and animal factors related to the relinquishment of dogs and cats in 12 selected animal shelters in the United States. Journal of Applied Animal Welfare Science, 1, pp. 207-226.

Sanders, C. (2003). Understanding Dogs: Caretakers' Attributions of Mindedness in Canine-Human Relationships. Journal of Contemporary Ethnography, 22, pp. 205

Saville-Troike, M. (2003). The Ethnography of Communication. Malden, MA: Blackwell Publishing Ltd.

Schultz, W. (1924). The Humane Movement in the United States, 1910-1922. New York: Columbia University Press

Serpell, J. (2000). Creatures of the Unconscious: Companion Animals as Mediators. Podberscek, L., Paul, E., Serpell, J. (Eds.) in Companion Animals \& Us, Exploring the relationships between People 7 Pets. pp. 108-119. New York: Cambridge University Press

Serpell, J. (1999). An Alternative History of Western Humaneness in F. R. Ascione \& P. Arkow (Eds.) Child Abuse, Domestic Violence and Animal Abuse: Unlinking the Circles of Compassion for Prevention and Intervention pp. 38-48 Indiana: Perdue University Press

Serpell, J. (1989). In the Company of Animals: A Study of Human-Animal Relationships. Basil Blackwell: New York. 
Spitzberg, B. H., \& Cupach, W. R. (1998). The dark side of close relationships. Mahwah, N.J: Lawrence Erlbaum Associates.

Taylor, N. (2004) In It for the Nonhuman Animals: Animal Welfare, Moral Certainty, and Disagreements. Society \& Animals, 12(4), pp. 317-339.

Taylor, N. (2007). "Never an It:" Intersubjectivity and the creation of animal personhood in Animal Shelters. Qualitative Sociology Review Vol III, Issue 1 pp. $59-73$

Trenholm, S. \& Jensen, A. (2007). Interpersonal Communication $\left(6^{\text {th }}\right.$ Ed.) Oxford University Press

Trenholm, S. \& Jensen, A (1996). Interpersonal Communication Wadsworth: Belmont

Wolfe, C. (2009) Human, All too Human "Animal Studies" and the Humanities. PMLA Vol. 124, Number 2, pp. 564-575 (12) 


\section{Appendix A.}

\section{Assumptions and Biases}

\section{Biases}

- I believe cities should have public services like the humane society in order to take care of the animal population.

- I believe most employees and volunteers treat animals in their care with respect and work at shelters because they genuinely like animals

\section{Prejudices}

- I have utilized the services of non-profit animal services in the past.

- I have so far had overall positive experiences in dealing with public animal services and employees.

\section{Expectations}

- I expect to find well-maintained facilities.

- I expected to find the Oregon Humane Society easy for the urban population of Portland to utilize.

\section{Values}

- I value companion animals.

- I believe in euthanasia for terminally ill/aged companion animals.

- I am currently the owner of a dog and grew up with a dog adopted from a shelter. 


\section{Appendix B.}

\section{Informed Consent}

\section{Animal Shelter Communication}

You are invited to participate in a research study conducted by Sara Kaufman from Portland State University, Department of Communication. The researcher hopes to better understand the various experiences of staff and volunteers working within a Pacific Northwest animal shelter in relation to companion animals. This study is being conducted in partial fulfillment of the requirements for a master's degree and is under the supervision of Dr. Susan Poulsen at PSU. You have been selected as a possible participant in this study because you are a volunteer or staff member at the Oregon Humane Society.

If you decide to participate, you will be asked about your experiences working at the Oregon Humane Society in interview format. The interview is expected to last about an hour, and will be audio-recorded. While participating in this study, it is possible that you may become a little uncomfortable when discussing your work with shelter animals or OHS. Your name will not be connected with your interview, and your responses will not be relayed to anyone at OHS. I will identify you by a pseudonym only, and this will be used instead of your real name. Any written accounts of the conversation will have your name changed. You can change your mind about your participation at any time before or during the conversation and can withdraw.

During the interview, please use an alias when you name people who work with OHS. You do not have to talk about anything you are uncomfortable with, and any question may be skipped. The audio recordings and any notes taken will be stored in a secure place, with access restricted to qualified researchers. You may not receive direct benefit from taking part in this study, but the study may help to increase knowledge, which may help others in the future. Your participation is voluntary. You do not have to take part in this study, and it will not affect your relationship with Portland State University or the Oregon Humane Society. You may withdraw from this study at any time without affecting your relationship with Portland State University or the Oregon Humane Society.

If you have any concerns or problems about your participation in this study, or your rights as a research participant, please contact the Human Subjects Research Review Committee, Office of Research and Sponsored Projects, 600 Unitus Bldg., Portland State University, (503) 725-4288/1877-480-4400. If you have any questions about the study itself, contact Sara Kaufman at duransd@gmail.com or (925) 683-3808

Your signature indicates that you have read and understood the above information and agree to take part in this study. Please understand that you may withdraw your consent at any time without penalty, and that signing, you are not waiving any legal claims, rights or remedies. The researcher will provide you with a copy of this form for your own records. 


\section{Appendix C.}

\section{Letter/E-mail Requesting Participation}

\section{Dear [Participant Name]}

This study seeks to better understand the various experiences of staff and volunteers working within a Pacific Northwest animal shelter in relation to companion animals.

\section{The Process:}

I will ask you to sign a release before the interview starts that indicates you have given me permission to record our conversation. Your identity remains confidential, I will identify you by a pseudonym only, and this will then be used instead of your real name. Any written accounts of the conversation will have your name changed. You can change your mind about your participation at any time before or during the conversation and can withdraw. Thank you for considering participation in this study. 


\section{Appendix D.}

Interview Guide

\begin{tabular}{|l|l|l|}
\hline Date: & Name: --- & Pseudonym: \\
\hline Start Time: & End Time: & Place: \\
\hline
\end{tabular}

(Thanks again for participating, I will turn on the audio recorder now)

1. Could you introduce yourself?

2. How long have you worked at OHS?

3. How did you become involved with OHS?

4. What has your experience been like working at OHS?

5. If you were talking with a friend, how would you describe what you do at OHS?

6. Tell me about a typical day for you at OHS?

7. Is there something you enjoy the most about working at OHS?

[probe: why?]

8. Is there something you enjoy the least?

[probe: why?]

9. Can you tell me about some experiences you've had working with companion animals at OHS?

10. Can you tell me about a companion animal at OHS that has make an impression on you?

[probe: why, how?]

11. Have your feelings about companion animals been affected by working with them at OHS?

[probe: in what ways?]

12. How would you describe the companion animals you work with at OHS to someone?

13. Are there certain words or phrases that people use at OHS?

[probe: Could you tell me a little more about some words I've heard around OHS]

14. Do you have any animals at home?

[probe: if you do, tell me about them]

15. Is there anything else you'd like to say or think I have missed?

16. Is there someone you know of who works or volunteers for OHS that would be helpful for me to talk with about this subject?

(for sampling purposes) 
Appendix E.

Glossary of Terms

ACT: Animal Care Technician

Bond: A connection between a human and animal, or animal and animal

Bonded Pair: Dogs who have lived most of their lives together and shelter staff does not adopt them out separately.

Cattery: Shelter area where cat kennels are housed

Colony Rooms: Rooms within the cattery where groups of three to eight cats are housed.

Euthanasia: humanely putting an animal to death.

Foster Care: Volunteers that care for a shelter animal in their home until it is ready to be adopted. These can include for example, pregnant or nursing cats, or an animal recovering from illness.

"Get Acquainted Room:" Room in the cattery where the public can spend time with a shelter cat.

Hoarder: A person(s) who has too many animals to properly care for "In the back:" Area within shelter where shelter workers are permitted, not the public Kennels: areas where dogs are housed, name of cat cages Kennel Cards: printed material either on the front side of the kennel or back side that contains information about ideal home or personality of the shelter animal.

Lassoing: Leashing a dog "Meet \& Greet Room:" Room in the dog kennels where the public can spend time with a shelter dog. 
OHS Alumni: Adopted animal once a shelter animal at OHS

On Supportive Care: Indicates a cat has kennel cough

Pet Meet: bringing a potential adopter's current companion animal to the shelter to "meet" a shelter animal in order to see if they get along.

Production: Routine, set time that dogs are walked.

Returns: Animals that have been adopted and returned to the shelter

Save Rate: Number of animals that have been adopted out from OHS or sent to another rescue/shelter

TTouch (Tellington Touch): A method of touch developed by Linda Tellington Jones that uses light pressure on skin to release tension of shelter animals. In the shelter, a goal of TTouch was to relax animals to make them more adoptable.

"Up front:" Area within the shelter where the public is permitted. 


\section{Appendix F.}

\section{Visual Glossary}

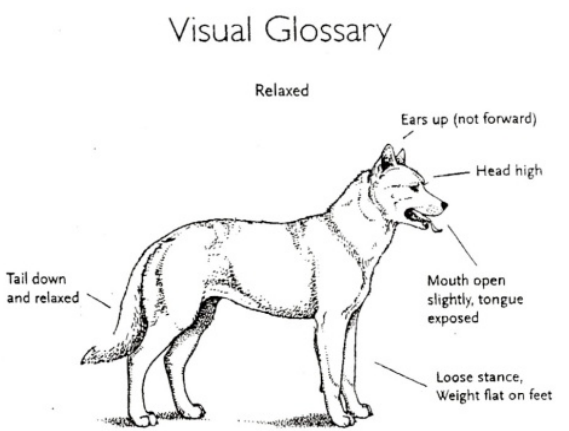

This array of signals communicates a relaxed, reasonably content dog who is unconcerned and unthreatened by any activities going on in its immediate en vironment.

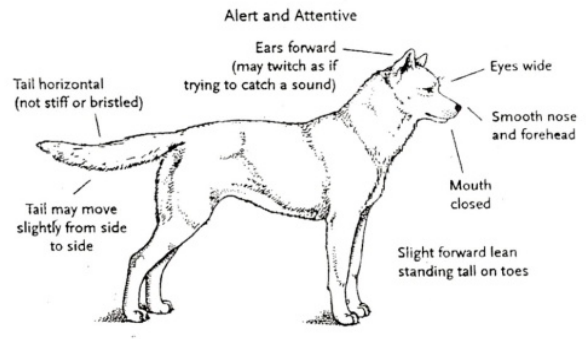

When something of interest is encountered or enters the environment, these signals communicate that attention is now being paid to them and the dog has entered a state of alertness.

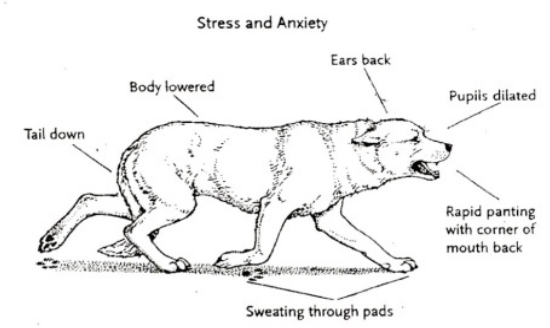

This is the pattern of signals which communicate that a dog is under stress. The source of the stress may be social or environmental, and the signals are not being specifically addressed to any other individual.

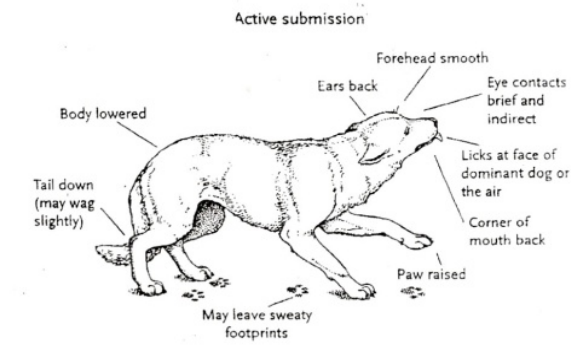

This pattern of signals communicates that the dog is somewhat fearful and is offering signs of submission. Most of these signars are designed to pacify the individual who is of higher social status in order to avoid any further chalienges

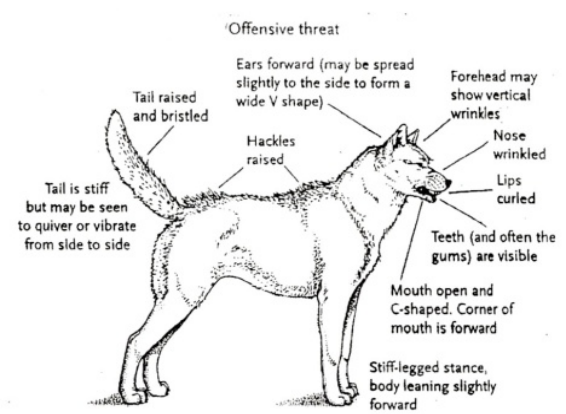

These signais are given by a very confident animal, who is com. municating both its social dominance and threatening aggression if it is

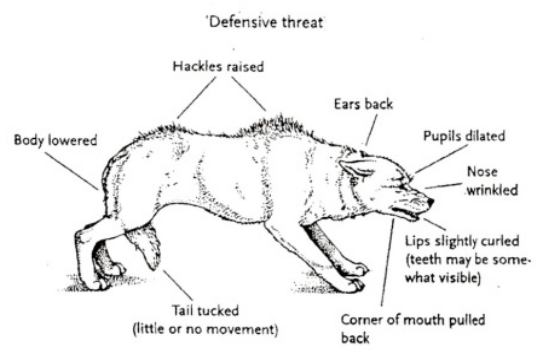

This set of signais communicates that the dog is frightened but is not submis. sive and may attack if pressed.
individual who is threatening.

Passive submission

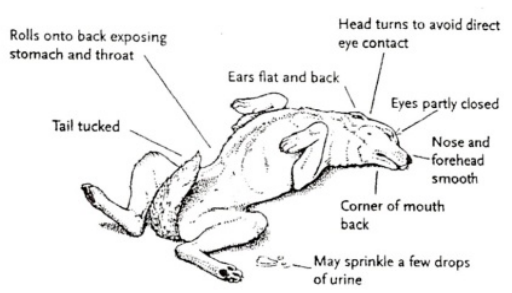

This pattern of signals indicates total surrender and submission. The dog indicates its lower status and grovels before the higher-ranking animal to pacify it and avoid confrontation

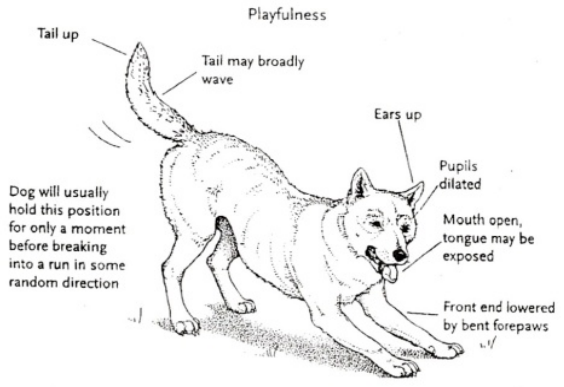

This is the basic invitation to play. It may be accompanied by excited barking, or cate that any previous rough behavior was not meant as a hreat: mark" to ind 